\title{
A Computational Model for Redundant Human Three- Dimensional Pointing Movements: Integration of Independent Spatial and Temporal Motor Plans Simplifies Movement Dynamics
}

\author{
Armin Biess, ${ }^{1}$ Dario G. Liebermann, ${ }^{3}$ and Tamar Flash ${ }^{2}$ \\ Departments of ${ }^{1}$ Mathematics and ${ }^{2}$ Computer Science and Applied Mathematics, Weizmann Institute of Science, 76100 Rehovot, Israel, and ${ }^{3}$ Department of \\ Physical Therapy, Stanley Steyer School of Health Professions, Sackler Faculty of Medicine, Tel Aviv University, 69978 Ramat Aviv, Israel
}

\begin{abstract}
Few computational models have addressed the spatiotemporal features of unconstrained three-dimensional (3D) arm motion. Empirical observations made on hand paths, speed profiles, and arm postures during point-to-point movements led to the assumption that hand path and arm posture are independent of movement speed, suggesting that the geometric and temporal properties of movements are decoupled. In this study, we present a computational model of 3D movements for an arm with four degrees of freedom based on the assumption that optimization principles are separately applied at the geometric and temporal levels of control. Geometric properties (path and posture) are defined in terms of geodesic paths with respect to the kinetic energy metric in the Riemannian configuration space. Accordingly, a geodesic path can be generated with less muscular effort than on any other, nongeodesic path, because the sum of all configuration-speed-dependent torques vanishes. The temporal properties of the movement (speed) are determined in task space by minimizing the squared jerk along the selected end-effector path. The integration of both planning levels into a single spatiotemporal representation simplifies the control of arm dynamics along geodesic paths and results in movements with near minimal torque change and minimal peak value of kinetic energy. Thus, the application of Riemannian geometry allows for a reconciliation of computational models previously proposed for the description of arm movements. We suggest that geodesics are an emergent property of the motor system through the exploration of dynamical space. Our data validated the predictions for joint trajectories, hand paths, final postures, speed profiles, and driving torques.
\end{abstract}

Key words: forward control strategies; point-to-point arm movements; geodesics; minimal effort; minimum jerk; minimum torque change

\section{Introduction}

A pointing movement toward a target in three-dimensional (3D) space defines a highly redundant task at the geometric, kinematic, and dynamic levels of control. An infinite number of possible hand paths can be selected for moving the hand to the target, and an infinite set of possible arm postures may be attained for every fixed hand location in 3D space. In particular, many arm configurations may be adopted at the end of the movement. Moreover, different speed profiles may be chosen along a given hand path, and diverse patterns of muscle activation may generate the same driving torques that ultimately move the hand toward the target.

The rules that the CNS applies to the control of movements are, in fact, poorly understood. One major difficulty results from

Received 0ct. 4, 2006; revised Aug. 13, 2007; accepted Aug. 14, 2007.

This work was supported in part by the Minerva Foundation, Germany, and in part by the DIP (German-Israel Project Cooperation). A.B. acknowledges support from a Dov Biengun research fellowship and thanks Dr. J. Reingruber for stimulating discussions. T.F. is the incumbent of the Dr. Hymie Moross Professorial Chair.

Correspondence should be addressed to Armin Biess, Department of Mathematics, Weizmann Institute of Science, 76100 Rehovot, Israel. E-mail: armin.biess@weizmann.ac.il.

D01:10.1523/JNEUROSCI.4334-06.2007

Copyright $\odot 2007$ Society for Neuroscience ～0270-6474/07/2713045-20\$15.00/0 the fact that the planning and control strategies cannot be directly accessed, and only some kinematic and dynamic movement properties can be measured under well defined experimental conditions. Invariant kinematic and dynamic properties observed during movement have provided some important insights into possible motor control strategies used by the CNS but led to a large number of different and apparently incompatible models (Hermens and Gielen, 2004).

Most existing models are based on the assumption that movements are planned before their execution. For example, it has been proposed that arm movements are planned in terms of hand Cartesian coordinates by maximizing motion smoothness (Flash and Hogan, 1985) or in terms of intrinsic joint coordinates by minimizing the squared change of joint torques (Uno et al., 1989; Nakano et al., 1999; Wada et al., 2001) and by minimizing peak value of kinetic energy (minimum peak work) (Soechting et al., 1995). In addition, stochastic models have been proposed assuming that the inherent noise in the motor system is minimized (Harris and Wolpert, 1998) or that the noise is optimally distributed among different degrees of freedom (Todorov and Jordan, 2002). 
Experimental observations suggest that hand paths and arm postures are invariant with respect to the scaling of movement speed (Flash and Hollerbach, 1982; Boessenkool et al., 1998; Nishikawa et al., 1999) and change in external forces (Atkeson and Hollerbach, 1985; Flanders et al., 2003). It has been shown that geometrically defined movement features can be acquired by the human motor system (Sosnik et al., 2004), before the specification of kinematic attributes such as movement speed (Torres and Zipser, 2002, 2004). It is thus conceivable that separate planning constraints may be imposed at the geometric and temporal levels. Both levels may subsequently be integrated into a complete spatiotemporal representation.

The present study aimed at developing a model of the motionplanning strategy underlying the generation of $3 \mathrm{D}$ pointing movements. The model is based on Riemannian geometry and accounts for hand paths, final arm postures, hand-speed profiles, and driving torques. It results in arm movements along geodesic paths that require less muscular effort than on any other, nongeodesic path, while maximizing smoothness. Our model thus reconciles different existing computational approaches into a single framework.

\section{Materials and Methods}

Theoretical background

A possible interpretation for the experimentally observed invariance of arm posture and hand path with changes in speed is the decoupling of geometric and temporal aspects in the trajectory-planning process of $3 \mathrm{D}$ arm movements. The objective of the computational model presented here consists of the prediction of movement patterns as resulting from a model that assumes that the geometric and temporal levels are independently planned, and the comparison of the predicted movements with experimentally observed trajectories. In the first stage, the geometrical properties of the movement as expressed by the joint-angular path in configuration space are determined. In the second stage, the temporal features of the movement in terms of hand speed are selected. In the third and final stage, the geometric and temporal features of the movement are integrated together into a unique spatiotemporal representation of the movement.

The approach for selecting the geometric properties of the movement is based on Riemannian geometry. Most readers will be familiar with Euclidean geometry, in which the squared distance between two nearby points that are separated by the vector $d x=\left(d \mathrm{x}_{1}, d \mathrm{x}_{2}, d \mathrm{x}_{3}\right)^{T}$ is given by

$$
d s^{2}=d x_{1}^{2}+d x_{2}^{2}+d x_{3}^{2},
$$

or written alternatively using a vector notation as

$$
d s^{2}=\boldsymbol{d} \boldsymbol{x}^{T} \boldsymbol{d x} .
$$

One also recalls that the shortest distance between two points in Euclidean space is a straight line. A train analogy is useful to further develop our basic model assumptions. We first observe that for a train, the speed is in principle independent of the shape of the specific railway track, similar to the decoupling of movement path from movement speed. We next consider the forces that act on the train in a hilly landscape. One might ask the following question: What is the optimal geometrical path between two stations A and B for laying a railway track in terms of forces that act on the train? This problem can be addressed by passing from Euclidean to Riemannian geometry.

In Riemannian geometry, the distance relationship is generalized with a metric tensor $g(\boldsymbol{x})$ that encodes the geometry of the hilly landscape locally near the point $\boldsymbol{x}$. The squared distance is given by

$$
d s^{2}=\boldsymbol{d} \boldsymbol{x}^{T} g(\boldsymbol{x}) \boldsymbol{d x} .
$$

Thus, Euclidean 3D space is a special case of a Riemannian manifold in which

$$
g=\left(\begin{array}{lll}
1 & 0 & 0 \\
0 & 1 & 0 \\
0 & 0 & 1
\end{array}\right)
$$

The optimal path for the train is given by the straightest possible path through the hilly landscape, because for this path the centripetal forces acting on the train are minimized. The determination of the straightest path is a standard problem in Riemannian geometry and results in the computation of the geodesic path connecting station A with B.

In the following paragraphs, we use Riemannian geometry to model human pointing movements in space. Using the previous analogy, we identify the arm with the train and the hilly landscape with the Riemannian configuration space. Thus, we are interested in the answers to the following questions: What is the optimal geometric path of the arm between a given initial and final arm posture in configuration space in terms of forces acting on the arm? What might be the optimal speed for the arm to move along the chosen path?

Detailed mathematical analysis. To further elaborate on these ideas, we have to provide some mathematical tools, which we present next. The configuration space of the arm, $Q$, defines a Riemannian manifold when endowed with a positive definite and symmetric metric tensor $g$ with components $g_{i j},(i, j=1, \ldots, n)$. A point in configuration space is denoted by $\boldsymbol{q}$ with (local) coordinates $\boldsymbol{q}=\left(q^{1}, q^{2}, \ldots, q^{n}\right) \in Q$. Distances in arm configuration space are defined as in Equation 3, which can alternatively be written in terms of the coordinates as

$$
d \sigma^{2}=\sum_{i=1}^{n} \sum_{j=1}^{n} g_{i j}(\boldsymbol{q}) d q^{i} d q^{j},
$$

where we have used the letter $\sigma$ for distance in the Riemannian manifold, and the letter $s$ is preserved in the following for distance in Euclidean space (Eq. 1). We make use in the following of the summation convention stating that repeated lower and upper indices imply a summation from 1 to $n$; thus, Equation 5 can be rewritten as $d \sigma^{2}=g_{i j}(\boldsymbol{q}) d q^{i} d q^{j}$. The metric tensor is symmetric, $g_{i j}=g_{i j}$, and assumed to be positive definite; i.e., it is $g_{i j} q^{i} q^{j}>0$ for all $\boldsymbol{q} \neq 0$, and thus it is invertible. The inverse of the metric tensor $g^{-1}$ has components $\left(g^{-1}\right)_{i j}=g^{i j}$ with

$$
g^{i k} g_{k j}=\left\{\begin{array}{ll}
1 & i=j \\
0 & \text { otherwise }
\end{array} .\right.
$$

In the Riemannian manifold, $(Q, g)$, the length of a curve $\gamma:[a, b] \rightarrow Q$ is defined as the line integral

$$
L(\gamma)=\int d \sigma=\int_{a}^{b} \sqrt{g_{i j} \frac{d q^{i} d q^{j}}{d \lambda d \lambda} d \lambda,}
$$

where $\lambda$ is an arbitrary parameter. Another quantity that is used in the following is the energy of a curve, which is defined as

$$
E(\gamma)=\frac{1}{2} \int_{a}^{b} g_{i j} \frac{d q^{i} d q^{j}}{d \lambda d \lambda} d \lambda .
$$

The name "energy" derives from the similarity of the integrand to the kinetic energy of a physical system. However, it is important to note that the energy of a curve is a geometrical quantity.

The curves that correspond to extremal paths in a Riemannian manifold are called geodesics. Geodesics are curves that are locally of minimal length. This means that any two points that are close enough are connected along the geodesics by the shortest possible path. It is not difficult to prove (do Carmo, 1992) that a curve of minimal length also minimizes the energy function (Eq. 8).

A necessary condition for an extremum of the energy function (Eq. 8), and thus for the length (Eq. 7), follows from the calculus of variation in the form of the Euler-Lagrange equations. The Euler-Lagrange equations applied to Equation 8 lead to the geodesic equation $(i=1, \ldots, n)$ 


$$
g_{i j} \frac{d^{2} q^{j}}{d \lambda^{2}}+\Gamma_{i j k} \frac{d q^{j} d q^{k}}{d \lambda d \lambda}=0, i=1, \ldots, n,
$$

where the Christoffel symbols of the first kind are defined by

$$
\Gamma_{i j k}=\frac{1}{2}\left(\frac{\partial g_{i j}}{\partial q^{k}}+\frac{\partial g_{i k}}{\partial d q^{j}}-\frac{\partial g_{k j}}{\partial q^{i}}\right), i, j, k=1, \ldots, n .
$$

We can rewrite Equation 9 using the "standard form" by multiplying it with the inverse of the metric to obtain

$$
\frac{d^{2} q^{i}}{d \lambda^{2}}+\Gamma_{j k}^{i} \frac{d q^{j} d q^{k}}{d \lambda d \lambda}=0, i=1, \ldots, n,
$$

where the Christoffel symbols of the second kind are defined by $\Gamma_{j k}^{i}=$ $g^{i l} \Gamma_{j k}$.

It is important to note that a geodesic curve is not only defined by the shape of its path, but also by its parameterization. It can be easily shown by using the geodesic Equation 11 that $d \sigma / d \lambda=$ const, and thus, the parameter $\lambda$ is linearly related to the arc length $\sigma$; i.e., it is $\lambda=a \sigma+b$ with some constants $a$ and $b$. A parameter $\lambda$ with this property is called affine parameter, and thus geodesic curves are parameterized with respect to an affine parameter. In particular, the arc length itself defines an affine parameter $(\lambda=\sigma)$ [the minimization of the energy function (Eq. 8) leads automatically to the parameterization of the geodesic path in terms of an affine parameter].

Of course, nothing prevents us from choosing any other, nonaffine parameterization (e.g., time $t$ ) along the path. However, such a choice implies that the geodesic Equation 11 is not satisfied. This can be shown if we define a new curve parameterization by the functional relation $t=$ $f(\sigma)$, where $\sigma$ is the arc length in configuration space. Then the derivatives are related according to the chain rule by

$$
\frac{d}{d \sigma}=f^{\prime} \frac{d}{d t^{\prime}}, \frac{d^{2}}{d \sigma^{2}}=f^{\prime} \frac{d}{d t}+f^{\prime 2} \frac{d^{2}}{d t^{2}}
$$

where a prime denotes differentiation with respect to $\sigma$. With the new parameterization, the geodesic equation in standard form becomes

$$
\frac{d^{2} q^{i}}{d t^{2}}+\Gamma_{j k}^{i} \frac{d q^{j} d q^{k}}{d t d t}=-\frac{f^{\prime \prime} d q^{k}}{f^{\prime 2} d t}, \quad i=1, \cdots, n .
$$

The left side of Equation 13 defines the acceleration in curvilinear coordinates and the term on the right side can be interpreted as a generalized force. For $t$ to be an affine parameter, $f^{\prime \prime}$ must vanish; i.e., $\sigma$ and $t$ must be linearly related or $\mathrm{d} \sigma / \mathrm{d} t=$ const. In this case, the force term vanishes, and Equation 13 transforms again into the geodesic equation (Eq. 11). Because the expression $\mathrm{d} \sigma / \mathrm{d} t$ defines a speed (if $t$ defines time), we conclude that geodesic paths are force-free paths of constant speed. Note that Equations 11 and 13 describe the same geodesic path but not the same geodesic curve.

To close this section, it is instructive to evaluate some of the above expressions in Euclidean geometry with coordinates $\boldsymbol{x}=\left(x_{1}, x_{2}, x_{3}\right)^{T}$ and a metric defined by Equation 4. For example, the energy of the curve is given by

$$
E(\gamma)=\frac{1}{2} \int_{a}^{b}\left|\boldsymbol{x}^{\prime}(\lambda)\right|^{2} \mathrm{~d} \lambda
$$

The geodesic equation (Eq. 11) transforms to $\boldsymbol{x}^{\prime \prime}(\lambda)=0$, leading to the well known result that geodesic paths of Euclidean space are straight lines.

Implications for human trajectory formation. How are these general mathematical considerations related to the model presented in the following sections? Our computational model assumes that the CNS adopts geodesic paths at the geometrical level as part of the trajectory-planning process. It will be shown in the following that geodesic paths with respect to a suitably chosen metric in configuration space simplify the arm dynamics significantly. It is this link between geometry and dynamics that makes the use of Riemannian geometry so attractive. We then hypothe- size that at the temporal level, the CNS selects a speed profile along the hand path in Euclidean task space, which in turn induces a nonconstant speed in configuration space $(d \sigma / d t \neq 0)$. The latter corresponds to a reparameterization of the geodesic paths in terms of a nonaffine parameter. This reparameterization does not change the shape of the geodesic path, but leads to a movement that is not force-free, and therefore, torques at the joints are required to drive the arm toward its final configuration. We will show in the following that the torques are reduced along geodesic paths, because the sum of all configuration-speed-dependent torques vanishes, whereas the remaining driving torques are to firstorder approximation linearly related to the hand acceleration. It is hypothesized in this paper that these paths are an emerging property of the system that seeks to reduce muscular efforts possibly based on proprioceptive feedback.

The next sections present the computational model. First, the forward and inverse kinematics of an arm with four degrees of freedom (DOFs) are presented as necessary prerequisites for the formulation of the computational model. This is followed by the details of the computational model and the description of the experimental protocol.

\section{Forward kinematics}

The human arm is approximated as a linkage of rigid bodies, and an arm configuration is parameterized by four joint angles $q:=(\theta, \eta, \zeta, \phi)^{T} \in Q$, where we follow a parameterization of the arm configuration given in Soechting et al. (1995). Q denotes the configuration space. The first three angles describe the rotation around an ideally spherical shoulder joint, namely, the elevation angle $\theta$, the azimuthal angle $\eta$, and the humeral angle $\zeta$, whereas the flexion angle $\phi$ determines the rotation around the elbow joint (Fig. $1 \mathrm{~A}$ ). We neglected the three DOFs at the wrist, which is assumed to be fixated. The shoulder joint is located at the origin of the laboratory frame coordinate system (CS). Two fixed body frames, $\mathrm{CS}_{1}$ with axes $x_{1}, y_{1}, z_{1}$ and $\mathrm{CS}_{2}$ with axes $x_{2}, y_{2}, z_{2}$, are attached to the upper arm and forearm segments, respectively, such that the $z$-axes are pointing along the longitudinal limb axes of the upper and forearm, and the $x$-and $y$-axes are in the transverse directions. In the zero configuration $[\boldsymbol{q}=(0$, $\left.0,0,0)^{T}\right]$, the arm is fully extended in the direction of the $(-z)$-axis, and the axis of the elbow joint is aligned with the $x$-axis. For the transformation of the arm from the zero configuration into an arbitrary configuration, the order of rotations has to be specified. In this work, an arm posture is defined as the sequence of the following rotations: (1) rotation around the $x_{1}$-axis by angle $\pi / 2$, (2) rotation around the $y_{1}$-axis by angle $\eta$, (3) rotation around the $x_{1}$-axis by angle $\theta-\pi / 2$, (4) rotation around the $z_{1}$-axis by angle $\zeta$, and (5) rotation around the $y_{2}$-axis by angle $\phi$, where the sign of the rotation angle is defined by the right-hand rule. The forward kinematics map defines the elbow and hand locations in the laboratory frame CS as a function of the joint angles; i.e., $\boldsymbol{x}_{e}=\boldsymbol{F}_{e}(\boldsymbol{q})$ for the elbow location and $\boldsymbol{x}_{h}=\boldsymbol{F}_{h}(\boldsymbol{q})$ for the hand location, where $\boldsymbol{x}_{e}=\left(x_{e}\right.$, $\left.y_{e}, z_{e}\right)^{T}$ and $\boldsymbol{x}_{h}=\left(x_{h}, y_{h}, z_{h}\right)^{T}$. For the chosen parameterization, we obtain

$$
\begin{aligned}
& x_{e}=-l_{u} \sin \eta \sin \theta, \\
& y_{e}=l_{u} \cos \eta \sin \theta, \\
& z_{e}=-l_{u} \cos \theta,
\end{aligned}
$$

and

$x_{h}=x_{e}-l_{f}[\sin \phi(\cos \zeta \sin \eta \cos \theta+\sin \zeta \cos \eta)+\cos \phi \sin \eta \sin \theta]$,

$y_{h}=y_{e}+l_{f}[\sin \phi(\cos \zeta \cos \eta \cos \theta-\sin \zeta \sin \eta)+\cos \phi \cos \eta \sin \theta]$,

$z_{h}=z e+l_{f}[\sin \phi \cos \zeta \sin \theta-\cos \phi \cos \theta]$,

where $l_{u}$ and $l_{f}$ are the upper arm and forearm lengths, respectively. It should be noted that the immobilization of the wrist motion, as was done for the experimental data analyzed here, implies that the hand and the wrist follow the same kinematics as one rigid link. Finally, for a given hand path, $\boldsymbol{x}_{h}=\boldsymbol{x}_{h}(\lambda)$, the change in hand path can be determined according to $\boldsymbol{x}_{h}^{\prime}(\lambda)=J_{h}(\boldsymbol{q}) \boldsymbol{q}^{\prime}(\lambda)$, where $\left(J_{h}\right)_{i j}=d \boldsymbol{F}_{h, i} / d q^{j}, i=1,2,3 ; j=1$, ..., 4 is the hand Jacobian, and a prime denotes differentiation with respect to $\lambda$, where $\lambda$ is a parameterization of the path. Similar relation- 

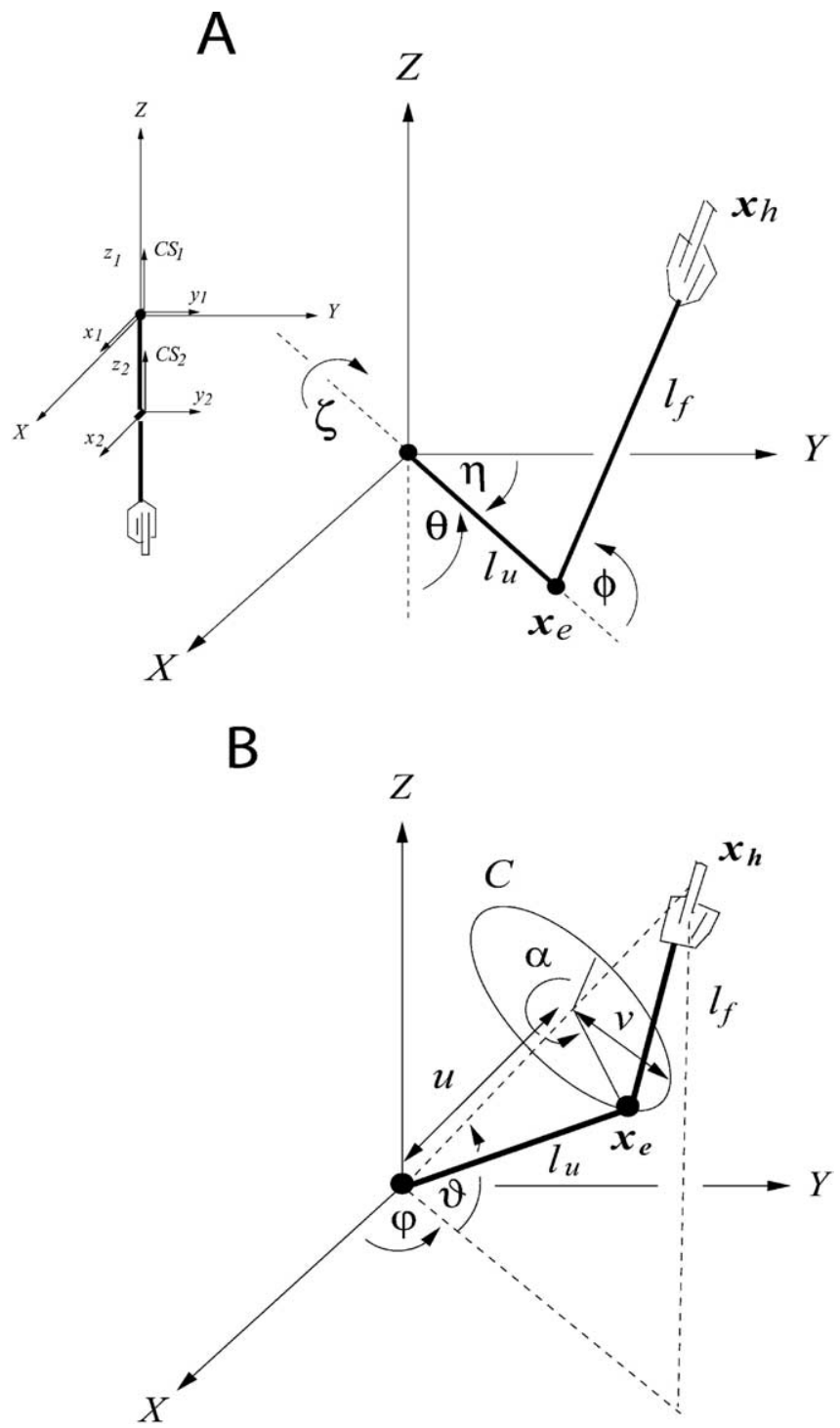

Figure 1. Forward and inverse kinematics. $\boldsymbol{A}$, General arm configuration defined by the three shoulder angles (elevation, $\theta$; azimuth, $\eta$; and torsion, $\zeta$ ) and the flexion angle $\phi$ at the elbow joint. The upper and forearm lengths are given by $I_{u}$ and $l_{f}$ respectively. The elbow and hand location, $\boldsymbol{x}_{e}$ and $\boldsymbol{x}_{h}$, are determined via the forward kinematics map for given joint angles. Inset, The zero arm configuration with the attached body-fixed coordinate systems, $\mathrm{CS}_{1}$ and $\mathrm{CS}_{2}$. $B$, Specification of the elbow location $\boldsymbol{x}_{e}$ for a given hand location $\boldsymbol{x}_{h}$ of an arm with four degrees of freedom used in the derivation of the inverse kinematics. All parameters are defined in Appendix B.

ships hold for the elbow path. The components of the elbow and hand Jacobian are given in Appendix A (the "hand Jacobian" defines the Jacobian of the whole arm system, whereas the "elbow Jacobian" defines the Jacobian of the upper arm segment).

\section{Inverse kinematics}

We determine next the inverse kinematic relation for the four DOF arm, which defines the joint-angular vector as a function of the hand location. As a result of the excess of one DOF in configuration space (four DOFs in configuration space and three DOFs in external task space for an immobilized wrist), there exists no unique map from hand to configuration space. Indeed, for a fixed hand location, the arm can still rotate around an axis going through the shoulder and the hand, implying that the elbow location is constrained to move on a circle around this axis (Hollerbach, 1985). Changes in the elbow position along the circle do not influence the hand location, and therefore, such changes do not contribute to the task goal. If we denote the rotation angle of the elbow around this axis with $\alpha \in[0,2 \pi)$, we can parameterize the elbow locations for a fixed hand location $\boldsymbol{x}_{h}$ as $\boldsymbol{x}_{e}=\boldsymbol{x}_{e}\left(\alpha ; \boldsymbol{x}_{h}\right)$ (Fig. $\left.1 B\right)$. An explicit expression for the elbow location in terms of the angle parameter $\alpha$ and the hand location $x_{h}$ is provided in Appendix B. Once the elbow and hand locations are fixed, the whole arm configuration is determined; i.e., there exists a relation of the form $\boldsymbol{q}=\boldsymbol{q}\left(\boldsymbol{x}_{e}, \boldsymbol{x}_{h}\right)$. An explicit calculation assuming that $\left|\boldsymbol{x}_{e} \times \boldsymbol{x}_{h}\right| \neq$ 0 and $\left|z_{e}\right|<l_{u}$ leads to

$$
\begin{aligned}
& \theta=\operatorname{acos}\left(\frac{-z_{e}}{l_{u}}\right) \\
& \eta=\operatorname{atan} 2\left(-x_{e}, y_{e}\right) \\
& \zeta=\operatorname{atan} 2\left(l_{u}\left(x_{e} y_{h}-x_{h} y_{e}\right), y_{e}\left(y_{e} z_{h}-y_{h} z_{e}\right)-x_{e}\left(z_{e} x_{h}-z_{h} x_{e}\right)\right) \\
& \phi=\operatorname{acos}\left(\frac{x_{h}^{2}+y_{h}^{2}+z_{h}^{2}-l_{u}^{2}-l_{f}^{2}}{2 l_{u} l_{f}}\right),
\end{aligned}
$$

where we defined the function $\operatorname{atan} 2(a, b):=\operatorname{atan}\left(\frac{a}{b}\right)-\operatorname{sign}(a)[1-$ $\operatorname{sign}(b)]\left(\frac{\pi}{2}\right)$. The inverse kinematic relation follows then by inserting the expression for the elbow locations, $\boldsymbol{x}_{e}=\boldsymbol{x}_{e}\left(\alpha ; \boldsymbol{x}_{h}\right)$, in the relationship expressing the joint-angular vector in terms of elbow and hand location, $\boldsymbol{q}\left(\boldsymbol{x}_{e}, \boldsymbol{x}_{h}\right)$.Thus, the inverse kinematic relation has the form $\boldsymbol{q}=\boldsymbol{q}\left(\alpha, \boldsymbol{x}_{h}\right)$. Note that the four components of the joint-angular vector are determined by the rotation angle $\alpha$ and by the three components of the hand location.

Obviously, not all rotation angles $\alpha \in[0,2 \pi)$ lead to a realizable arm posture. The subset of rotation angles that lead to postures inside the biomechanically admissible joint range are determined by using biomechanical joint-range models. We assume that only joint angles that satisfy the following inequalities lead to admissible arm configurations:

$$
\begin{aligned}
0 & \leq \theta(\alpha) \leq \pi, \\
-\frac{3}{4} \pi & \leq \eta(\alpha) \leq \frac{\pi}{3}, \\
\zeta_{\text {ext }}(\theta, \eta) & \leq \zeta(\alpha) \leq \zeta_{\text {int }}(\theta, \eta), \\
0 & <\phi<\pi,
\end{aligned}
$$

where the external and internal humeral rotation of the upper arm, $\zeta_{\text {ext }}$ and $\zeta_{\text {int }}$, describe the maximal clockwise and counterclockwise rotations, respectively, around the upper-arm axis as a function of upper-arm axis orientation (when viewed from the subject). The external and internal humeral rotations are derived from experimental data and are approximated by the curved surfaces shown in Figure 2 (Wang et al., 1998).

For each hand location in space, $\boldsymbol{x}_{h}$, the interval of possible rotation angles, $I\left(\boldsymbol{x}_{h}\right) \supset \alpha$, defines the arm posture constraints. The corresponding joint-angular vectors associated with realistic arm postures at hand location $x_{h}$ are thus specified by the one-parameter family of vectors $\boldsymbol{q}(\alpha)=\boldsymbol{q}\left(\boldsymbol{x}_{h}, \alpha\right)$ with $\alpha \in I\left(\boldsymbol{x}_{h}\right)$.

\section{Description of the computational model}

The implementation of the geometric and temporal levels in the trajectory planning process and the integration into a spatiotemporal representation are described in the following sections.

Geometric level: prediction of the joint-angular path in configuration space. The configuration space of the arm defines a Riemannian manifold when assigning a suitable (positive definite and symmetric) metric tensor in configuration space. A natural candidate for a metric in configuration space is defined by the (in general, non-Euclidean) kinetic energy metric $M$ (or manipulator inertia matrix in the robotic literature); i.e., we set $g_{i j}=M_{i j}, i, j=1, \ldots, 4$ (Biess et al., 2001). The components of the kinetic energy metric are given by

$$
M_{i j}(\boldsymbol{q})=\frac{\partial K(\boldsymbol{q}, \dot{\boldsymbol{q}})}{\partial \dot{q}^{i} \partial \dot{q}^{j}},
$$



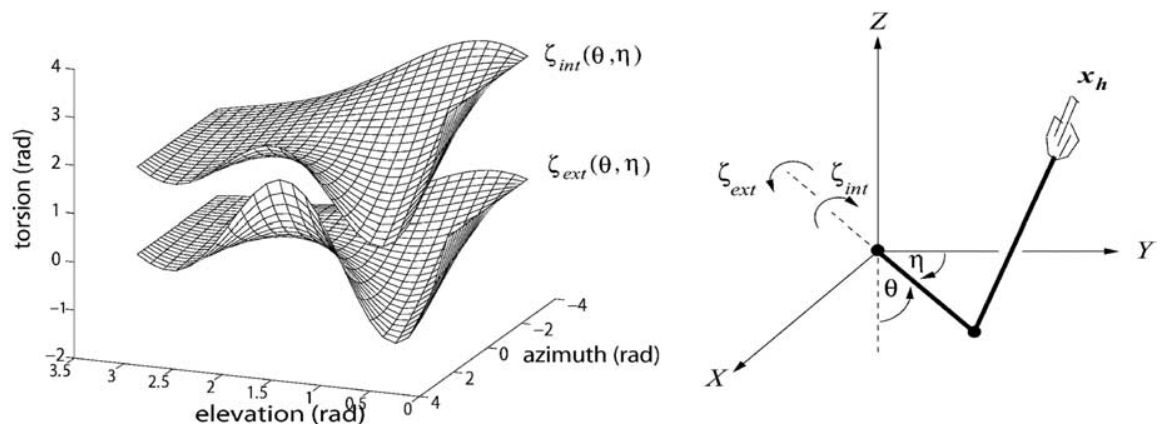

Figure 2. Definition and estimation of the external and internal humeral rotation, $\zeta_{\text {ext }}$ and $\zeta_{\text {int }}$, as a function of the upper-arm direction. The external and internal humeral rotation describe the maximal clockwise and counterclockwise rotations, respectively, around the upper-arm axis as a function of upper-arm axis orientation (when viewed from the subject). The external and internal humeral rotations are derived from experimental data and are approximated by the curved surfaces (Wang et al., 1998).

where $K$ is the kinetic energy of the arm and $q^{i}, i=1, \ldots, 4$, denote the components of the joint-angular vector $\boldsymbol{q}=(\theta, \eta, \zeta, \phi)^{T}$. The explicit form of the kinetic energy and the components of the kinetic energy metric for a four DOF arm are provided in Appendix C.

The kinetic energy metric depends on the current arm configuration and represents physically the instantaneous composite mass distribution of the whole arm linkage in the current arm configuration (Asada and Slotine, 1986). The use of this metric is motivated by studies of Soechting et al. (1995) and Flanders et al. (2003) that showed the significance of inertial properties of the whole arm for the selection of the arm configuration in $3 \mathrm{D}$ space. The observed variability of hand-path curvature, which depends on the location in the task space, makes the kinetic energy metric attractive for the description of 3D point-to-point movements. In addition, the kinetic energy metric is closely related to the dynamic equations of motion of the arm, as will be shown in Results. The line element associated with the kinetic energy metric is, according to Equation 5,

$$
d \sigma^{2}=M_{i j}(\boldsymbol{q}) d q^{i} d q^{j} .
$$

We can then compute the geodesic path for the kinetic energy metric between an initial and final configuration, $\boldsymbol{q}_{0}$ and $\boldsymbol{q}_{f}$, respectively. In this work, we define the affine parameter, $\lambda$, of the geodesic path as follows: if we denote with $\Sigma$ the total arc length of the path in configuration space between the initial and final configuration, we define the (dimensionless) affine parameter by the normalized arc length $\lambda=\sigma / \Sigma$, which then takes values between 0 and 1 . The geodesic equation for the kinetic energy metric is given as a result of Equation 9 by $\left(g_{i j}=M_{i j}\right)$

$$
M(\boldsymbol{q}) \boldsymbol{q}^{\prime \prime}+C\left(\boldsymbol{q}, \boldsymbol{q}^{\prime}\right) \boldsymbol{q}^{\prime}=0,
$$

where $\boldsymbol{q}^{\prime}=d \boldsymbol{q} / d \lambda$ and the components of, the Coriolis matrix, $C=\left(C_{i j}\right)$, $i, j=1, \ldots, 4$, are defined by

$$
C_{i j}=\Gamma_{i j k} \frac{d q^{k}}{d \lambda}=\frac{1}{2}\left(\frac{\partial M_{i j}}{\partial q^{k}}+\frac{\partial M_{i k}}{\partial q^{j}}-\frac{\partial M_{k j}}{\partial q^{i}}\right) \frac{d q^{k}}{d \lambda} .
$$

The specification of suitable boundary conditions is required for the solution of the geodesic equation (Eq. 31). We set

$$
\begin{aligned}
& \boldsymbol{q}(0)=\boldsymbol{q}_{0}, \\
& \boldsymbol{q}(1)=\boldsymbol{q}\left(\alpha ; \boldsymbol{x}_{f}\right), \alpha \in I\left(x_{f}\right) .
\end{aligned}
$$

For this choice of boundary conditions, the complete initial arm configuration, $\boldsymbol{q}_{0}$, and the final hand location, $\boldsymbol{x}_{h}=\boldsymbol{x}_{f}$, are specified. However, because of kinematic redundancy, the given final hand location corresponds to a one-parameter family of possible final arm configurations, namely, all the accessible arm configurations that are generated by a rotation around the axis connecting the shoulder joint and the final hand location. The final arm posture is thus not uniquely determined. Instead, it is an outcome of the optimization procedure described below.

We remark that the computation of the solution of the geodesic equation (Eq. 31), subject to the set of boundary conditions in Equations 33 and 34 , is not a trivial problem. Mathematically, it defines a nonlinear two-point boundary value problem. A numerical solution for the computation of the geodesics with a high degree of accuracy was developed and presented by Biess et al. (2006), and we refer the reader to this work for additional details.

We describe next the computational steps to determine the geometric prediction of the model in terms of the optimal joint-angular path in configuration space (Fig. 3). The configuration space $Q$ is visualized as a twodimensional surface. Each point in configuration space corresponds to an arm posture. The input to the model is given by the initial arm configuration, $\boldsymbol{q}_{0}$, and the final target location $\boldsymbol{x}_{f}$, where the latter corresponds to a oneparameter family of final arm configurations. To extract this set of final arm postures, we compute first the circle $C$ in task space, which contains the set of final elbow locations. The set of final elbow locations determines together with the fixed final hand location the set of possible final arm configurations. In configuration space, this set can be represented as a one-dimensional manifold. Next, we determine the set of admissible arm postures. For this purpose, the final elbow positions on the circle $C$ are discretized (by variation of the angle $\alpha$ in steps of $3^{\circ}$ ), and the final elbow locations inside $(\bullet)$ and outside $(O)$ of the biomechanically admissible joint range are derived from biomechanical joint-range models. These sets correspond in configuration space to subsets of the one-dimensional manifold. In the final step, we compute for each fixed value of the angle $\alpha \in I\left(\boldsymbol{x}_{h}\right)$ the geodesic path between the initial arm configuration, $\boldsymbol{q}_{0}$, and the final arm configuration, $\boldsymbol{q}_{f}(\alpha)=\boldsymbol{q}\left(\alpha ; \boldsymbol{x}_{f}\right), \alpha \in I\left(\boldsymbol{x}_{f}\right)$. The solution of Equation 31 subject to Equations 33 and 34 thus defines a one-parameter family of geodesics in configuration space, $\gamma_{\alpha^{*}}:[0,1] \rightarrow Q$, that all start at the same point but end at different points in configuration space. In extrinsic task space, this corresponds to a fixed initial arm posture, whereas many final postures may be compatible with the given final hand location. Among all geodesics that are compatible with the task constraints, we assume that the CNS selects the geodesic path with the minimal length in configuration space; i.e., we select the optimal path in joint configuration space as the geodesic path with the property

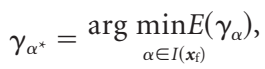

where the energy of the curve is given according to Equation 8 by

$$
E\left(\gamma_{\alpha}\right)=\frac{1}{2} \int_{0}^{1} \boldsymbol{q}^{\prime}(\lambda, \alpha)^{T} M(\lambda, \alpha) \boldsymbol{q}^{\prime}(\lambda, \alpha) d \lambda=\frac{1}{2} \int_{0}^{1}\left(\frac{d \sigma}{d \lambda}\right)^{2} d \lambda=\frac{1}{2} \sum^{2}(\alpha)
$$

and $\alpha=\alpha^{\star}$ is the rotation angle that minimizes the energy of the curve. $\Sigma(\alpha)$ denotes the arc length of path $\gamma_{\alpha}$ in configuration space. The optimal joint-angular path is thus determined by the geodesic path $\gamma_{\alpha^{*}}$ : $\boldsymbol{q}^{\star}(\lambda)=\boldsymbol{q}\left(\lambda, \alpha^{*}\right)$, and the optimal hand path in task space follows from the forward kinematics map. The temporal properties of the movement are determined by ascribing a speed profile along the hand path, as presented in the next section.

Temporal level: prediction of the speed profile in Euclidean task space. The timing of the movement is derived in task space, where distance is defined according to the Euclidean metric (Eq. 2). It is assumed that the speed profile along the hand path is determined by minimization of the squared jerk of the arc length $s$ of the hand path integrated over the movement time:

$$
C=\int_{0}^{T} \dddot{s}^{2} d t
$$


subjected to the boundary conditions

$$
\left.\begin{array}{l}
s(0)=0, \quad s(T)=S, \\
\dot{s}(0)=0, \quad \dot{s}(T)=0, \\
\ddot{s}(0)=0, \quad \ddot{s}(T)=0,
\end{array}\right\}
$$

where $T$ is the measured total movement duration, $S$ is the total Euclidean arc length of the hand path, and a dot denotes differentiation with respect to time. Note that Equations 37 and 38 correspond to a modified minimumjerk (MJ) model that is expressed in terms of the arc length along the hand path rather than in terms of the end-effector coordinates as in its original formulation. Remember that the solution of the original minimum-jerk model resulted in straight hand paths with a bell-shaped velocity profile. In the modified minimum-jerk model, we only determine the time course (speed) along an a priori defined path that can have any smooth shape.

The modified minimum-jerk model is motivated by the observation that speed profiles of point-to-point movements in $3 \mathrm{D}$ are roughly bell shaped (Atkeson and Hollerbach, 1985). This choice will be also motivated retrospectively when discussing the dynamical properties of the resulting movement. The optimal solution for position and speed is given by (Flash and Hogan, 1985)

$$
\begin{aligned}
& s^{\star}(t)=S \tau^{3}\left(10-15 \tau+6 \tau^{2}\right), \\
& v^{\star}(t)=\frac{15}{8}\left(\frac{S}{T}\right)[4 \tau(1-\tau)]^{2} .
\end{aligned}
$$

The total movement time is an input parameter taken from the measurements, whereas the total arc length of the hand path is not known a priori but can be computed from the joint-angular paths determined at the geometrical level. The arc length of the hand path in Euclidean task space follows from Equation 2 as

$$
\frac{d s}{d \lambda}=\left|\boldsymbol{x}^{\prime}(\lambda)\right|
$$

which leads to

$$
s(\lambda)=\int_{0}^{\lambda}\left|\boldsymbol{x}^{\prime}(\mu)\right| d \mu=\int_{0}^{\lambda}\left|J(\boldsymbol{q}(\mu)) \boldsymbol{q}^{\prime}(\mu)\right| d \mu,
$$

where we expressed the hand vector in terms of the joint-angular vector using the hand Jacobian. The total arc length of the hand path in task space follows then as $S=s(1)$. It should be noted that the arc length of the hand path as a function of path parameter is a monotonically increasing function $(d s / d \lambda>0)$ and thus can be inverted, leading to a function $\lambda=$ $\lambda(s)$.

The relation between the speed in configuration space, $\dot{\lambda}$, and the speed in task space, $\dot{s}$, is derived next. From Equation 41, we get

$$
\dot{\lambda}=\dot{s} \lambda^{\prime}(s),
$$

where a dot and a prime denote differentiation with respect to time and arc length $s$ of the hand path, respectively. For later purposes, we also derive the acceleration in configuration space, which follows by derivation of Equation 43 as

$$
\ddot{\lambda}=\ddot{s} \lambda^{\prime}(s)+\dot{s}^{2} \lambda^{\prime \prime}(s) .
$$

task space

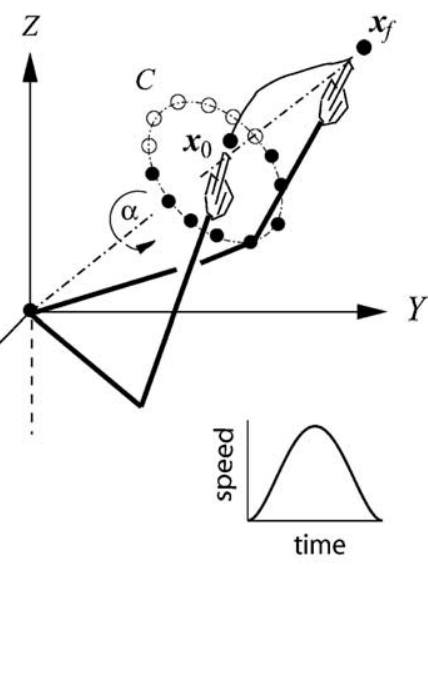

Figure 3. Description of the computational model in configuration space (left) and task space (right). The configuration space Qis is selected as the geodesic with minimal arc length in configuration space over all accessible final arm configurations. The optima hand path follows from the forward kinematics. A minimum-jerk speed profile is then assigned along the hand path, which determines the temporal properties of the movement.

Note that the kinetic energy of the arm is according to Equations 30 and 43 given by $(\sigma=\lambda / \Sigma)$

$$
K=\frac{1}{2} \dot{\sigma}^{2}=\frac{1}{2}\left(\sum \lambda^{\prime}(s)\right)^{2} \dot{\boldsymbol{s}}^{2}
$$

Equation 45 is used in Appendix D for the derivation of the minimumpeak kinetic energy (minimum peak work) model, which follows as an outcome of our computational model.

Spatiotemporal level: integration of the geometric and temporal levels. The spatiotemporal representation of the movement can be constructed from the selected motion patterns at the geometric and temporal levels by two successive transformations. First, the optimal joint-angular path, $\boldsymbol{q}^{*}(\lambda)$, is reparameterized with respect to the arc length of the hand path; i.e., $\boldsymbol{q}^{\star}(s)=\boldsymbol{q}^{\star}(\lambda(s))$. Then, the optimal time profile is imposed along the hand path, resulting in the optimal joint-angular trajectory, $\boldsymbol{q}^{\star}(t)=$ $\boldsymbol{q}^{\star}\left(s^{\star}(t)\right)$, which defines the optimal movement kinematics. For brevity, we refer in the following to the model with these temporal and geometrical features as the geodesic (GEO) model. The implications of the GEO model for the arm dynamics are presented in Results.

\section{Experiments}

Subjects. Four right-handed male volunteers (age range, 18-32 years) completed a series of natural unconstrained arm movements of two different types within a single session. None of the participants reported having any clinical symptoms or any history of motor, sensory, or neurological disorders. All participants gave their signed consent to participate in the experiment, as requested by the institutional ethics committee.

Procedure. Subjects sat in front of a projection screen (a parafrontal plane relative to their torso) with the shoulder at a distance of $1 \mathrm{~m}$. They were strapped to a chair by appropriate belts that minimized shoulder displacements and fixated the torso throughout the experiment. The height of the chair was adjusted such that the right shoulder was aligned 
with a fixed reference point in space. The subjects were naive to the purpose of the experiment. They were instructed to point toward visual targets that were randomly presented in different locations of the $3 \mathrm{D}$ task space. All experiments were performed under dimmed lighting conditions such that individuals were able to see their arm. Targets consisted of $5 \mathrm{~cm}$ balls that were projected on the screen. For this purpose, a 3D virtual reality was generated using stereovision. For additional details, we refer to Liebermann et al. (2006). The 3D positions of infrared emitting diodes (IREDs) were recorded at a rate of $100 \mathrm{~Hz}$ with an accuracy $<1$ mm using two motion tracking cameras (Optotrak; Northern Digital, Waterloo, Ontario, Canada). The IREDs were attached to the arm segments via exoskeletal metal frames. The center of the frame placed over the acromion (a triangle of $10.7 \mathrm{~cm}$ base and $11 \mathrm{~cm}$ height) was the assumed center of shoulder rotation. The center of the exoskeletal frame placed on the distal end of the upper arm ( $18.5 \mathrm{~cm}$ height, $16.2 \mathrm{~cm}$ width) was used to measure the elbow joint location. A third frame $(11.4 \mathrm{~cm}$ height, $11 \mathrm{~cm}$ width) was centered and attached to the wrist. The latter was braced to eliminate any rotations of the radiocarpal joint (i.e., supination-pronation movements were only possible by rotating the forearm about the radioulnar joint).

Experimental protocol. Data were collected during the performance of radial and frontoparallel movements. Radial movements were defined as the hand motion in the inward and outward directions relative to the body's longitudinal axis. In the radial condition, targets were presented at fixed distances of 0.6 and $0.8 \mathrm{~m}$ from the location of the shoulder center, at different heights. Pointing always started from a central position $(0.3$ $\mathrm{m}$ frontally and at $0 \mathrm{~m}$ horizontally and vertically relative to the shoulder). A set of radial movements consisted of 78 trials from the initial to the final targets and vice versa (forward and backward movements). Each set of movements was repeated five times, resulting in a total number of $78 \times 2 \times 5=780$ movement trials.

Frontal plane movements were defined as movements between targets lying on frontoparallel planes relative to the body. Targets were presented in four frontoparallel planes at fixed distances of $0.30,0.45,0.60$, and $0.75 \mathrm{~m}$ from the shoulder center. Each plane contained four initial targets and 12 final targets presented at different heights $(-0.4,0$, and $0.4 \mathrm{~m}$ relative to the shoulder). It is important to note that the end effector was not constrained to move in the virtual plane between an initial and a final target. A set was comprised of 24 pointing movements from a fixed initial target position to several final targets in the same frontal plane and their reversals. The initial target within one of the four planes was changed four times, and each set was repeated five times, resulting in a total number of $5 \times 16 \times 24=1920$ movements. In both movement conditions, the subjects could choose freely the initial arm configuration at the initial target.

Data analysis. During the data collection process, markers were not always visible to the cameras, and data were lost. However, only if more than one-third of the total number of samples obtained for the IRED markers were lost during a movement was the trial excluded. In all other cases, a data recovery process was initiated as follows. Geometric and temporal information was used for recovering data. If one marker of an exoskeleton at a certain time frame was missing, it could be simply restored by geometrical considerations based on the rigidity of the exoskeletons. If marker signals were lost over a whole interval of frames, where the maximal allowed contiguous missing segment was set to 15 frames $=$ $150 \mathrm{~ms}$, the missing markers were reconstructed by extrapolating from all visible markers before and after the missing time frames. Raw displacement data obtained from the markers were filtered (Butterworth, second order). The cutoff frequency was chosen depending on whether position, velocity, or acceleration data were generated (position: $6.0 \mathrm{~Hz}$; velocity: $5.5 \mathrm{~Hz}$; acceleration: $5.0 \mathrm{~Hz}$ ) (Giakas and Baltzopoulos, 1997). The movement onset and offset times are determined by optimally fitting (least-square error) a superposition of minimum-jerk speed profiles to the absolute hand velocity profile (Lee et al., 1997). Not more than two minimum-jerk submovements were needed to extract the movement onset and offset times. This procedure was only used to reconstruct the segments of the experimental speed profiles near the beginning and the end of the movement (approximately the segments of the speed profiles for which $v<0.15 v_{\max }$ ) and not to fit a minimum-jerk speed profile to the data.

The estimation of the elbow locations from the marker positions of the exoskeletons, which are attached to the limbs, is an essential part of the data analysis. Skin movements and random measurement noise lead to errors in the measured joint locations. In particular, errors occur in the localization of the elbow joint because of the difficulties in attaching the exoskeleton such that no relative motions between arm and grid occur while moving. However, a reliable elbow position is required for the determination of the joint angles and the length of the limbs. The estimation of the elbow position was composed of three steps. First, the marker coordinates were transformed to a coordinate system that moved with the upper arm. Second, an optimal estimate of the elbow location in the upper arm coordinate system was determined (Gamage and Lasenby, 2002). In this coordinate system, only the forearm moves, and the elbow location is a fixed point that can be determined by geometric methods. The estimated elbow locations in the shoulder-fixed coordinate system followed then by back-transformation.

\section{Results}

The results of the Riemannian approach were compared with the predictions of the Euclidean approach, where a Euclidean metric is assumed in task and configuration space. Thus, the metric $M(\boldsymbol{q})$ is replaced by the identity matrix. The geodesic paths in configuration space are then simply straight lines given by

$$
\boldsymbol{q}(\lambda)=\boldsymbol{q}_{0}+\lambda\left(\boldsymbol{q}_{\mathrm{f}}-\boldsymbol{q}_{0}\right) .
$$

This path is the optimal solution to the squared-joint derivative cost (SJD),

$$
C_{S J D}=\int_{0}^{1} \boldsymbol{q}^{\prime}(\lambda)^{\mathrm{T}} \boldsymbol{q}^{\prime}(\lambda) d \lambda .
$$

The temporal prediction for the SJD model was assumed to result from the minimum-jerk model for the arc length, as specified in Equations 37 and 38.

In addition, the results of the two previous models were compared with the predictions of the minimum torque-change (MTC) model. This model minimizes the squared change of joint torques integrated over the total movement time (Uno et al., 1989). Boundary conditions for the MTC model were set according to Equations 33 and 34, supplemented by zero joint-angular velocities and accelerations at the beginning and end of the movement. As for the geodesic model, the final arm posture was not predefined, but rather resulted as an outcome of the optimization. For the solution of the MTC model, an optimization method based on the parameterization of joint-angular trajectories was used (Biess et al., 2006).

Simulated and observed data are compared in this section. Results are presented in four subsections that addressed predictions at the geometric, temporal, spatiotemporal, and dynamic levels.

\section{Geometric level: hand paths and final postures}

Measured hand paths were persistently curved depending on the location in the workspace. However, the curvature was small for all types of movements. The curvature of the hand paths was assessed by evaluating a global curvature index defined as $\mathrm{CI}=$ $S / L$, where $S$ denotes the total arc length of the hand path and $L$ the Euclidean distance between the initial and the final targets. For straight hand paths, the curvature index is $\mathrm{CI}=1$, whereas for a semicircular path, the index is $\mathrm{CI}=\pi / 2 \approx 1.57$. The last columns of Figures 9 and 10 show the distributions of the curvature index for radial and frontoparallel movements, respectively. 
As can be seen from these figures, most of the movements result in quasi-straight hand paths with a curvature index $\mathrm{CI} \leq$ 1.05 .

Typical examples of the measured and predicted hand paths for the three models (GEO, SJD, and MTC) in the radial condition are shown in Figure 4 and in the frontal plane condition in Figure 5. Each row shows the projections of the three dimensional hand path of one movement into the $x y$-, $x z$-, and $y z$-planes. These randomly chosen examples from the data of our four subjects show that the MTC model consistently deviates from the observed paths in all three planar projections of the 3D hand movements. This was observed regardless of the movement condition or direction. In the same vein, it may be observed that the GEO and SJD models are close to each other. For the evaluation of the path predictions, a (global) hand-path deviation index (HPDI) based on the maximal value of the minimal Euclidean distances between measured and predicted paths was calculated. Such an index was used to quantify differences such as those observed in Figures 4 and 5. The measured path was divided into $M=40$ equidistant segments, which defined $M-1$ inner points. The minimal Euclidean distances from the inner points to the predicted path, $R_{i}, i=1, \ldots, M-1$, were determined, and the ratio of the maximal value $R=\max _{i=1, \ldots M-1}\left\{R_{i}\right\}$ and the distance between the initial and final targets $L$ was defined as the hand-path deviation index, HPDI $=R / L$ (Fig. $6 A$ ).

In all presented cases, the path predictions of the GEO model were closer to the measured hand paths, as indicated by the mean HPDI scores presented in Table 1, than the path predictions derived from the two other models. The paths resulting from the GEO and SJD models were similar, whereas the latter led to slightly larger HPDI values. In contrast, the hand paths resulting from the MTC model were strongly curved in most trials, and thus, large HPDI values were obtained for the MTC model.

In the present experiments, the arm was free to adopt any configuration among a large number of possibilities available during pointing to visual targets, in particular, at the final target. However, reproducible postural patterns were commonly observed within a limited set. The present model was designed to predict those arm postures that bring the hand to its final position by the shortest geodesics that connects the initial and the final arm posture in configuration space. The predicted versus the measured final joint angles of the shoulder are shown in Figure 7 for the radial movements and in Figure 8 for movements in a frontal parallel plane, together with the $R^{2}$ values for the GEO, SJD, and MTC models. The figures show that the largest deviations from the perfect fit, where all data points lie on the diagonal, occur for the MTC model, whereas the GEO and the SJD model lead to similar and less spread-out distributions around the regression line. The analysis of the $R^{2}$ values resulted in the highest score for the GEO model, although the torsional angles $\zeta$ were not always predicted successfully. For example, in the case of subject 1 in the radial condition and subject 3 in the frontal-plane condition, Figures 7 and 8 show respectively for each of these subjects and conditions that the torsion angle was not predicted very well. The origin of this large discrepancy for these subjects remained unclear.

To further evaluate the predicted final arm postures, a relative error measure of the form of the final posture deviation index (FPDI) was determined as follows. At a given target location, the arm can rotate around an axis going through the shoulder and the final hand location. The rotation angle around this axis is given by the angle $\alpha$ (Fig. $1 B$ ). The measured and predicted final arm postures can thus be defined by the angles $\alpha_{\exp }$ and $\alpha_{\text {pre }}$, respectively. The deviation index for each final arm posture follows then as the ratio of the absolute difference between the predicted and measured angles, $\Delta \alpha=\left|\alpha_{\exp }-\alpha_{\text {pre }}\right|$, to the total anatomically accessible range, $\Delta \alpha_{\text {tot }}=\left|I\left(\alpha ; \boldsymbol{x}_{f}\right)\right|$ at the final hand location $\boldsymbol{x}_{h}=$ $\boldsymbol{x}_{f}$. Note that the total anatomically accessible angular range depends on the hand location and can be estimated from biomechanical joint-range models (Fig. 6B). As shown in Table 1, the FPDI measures were smallest for the GEO model, followed by the predictions of the SJD cost. In comparison, large values were obtained for the MTC model. 

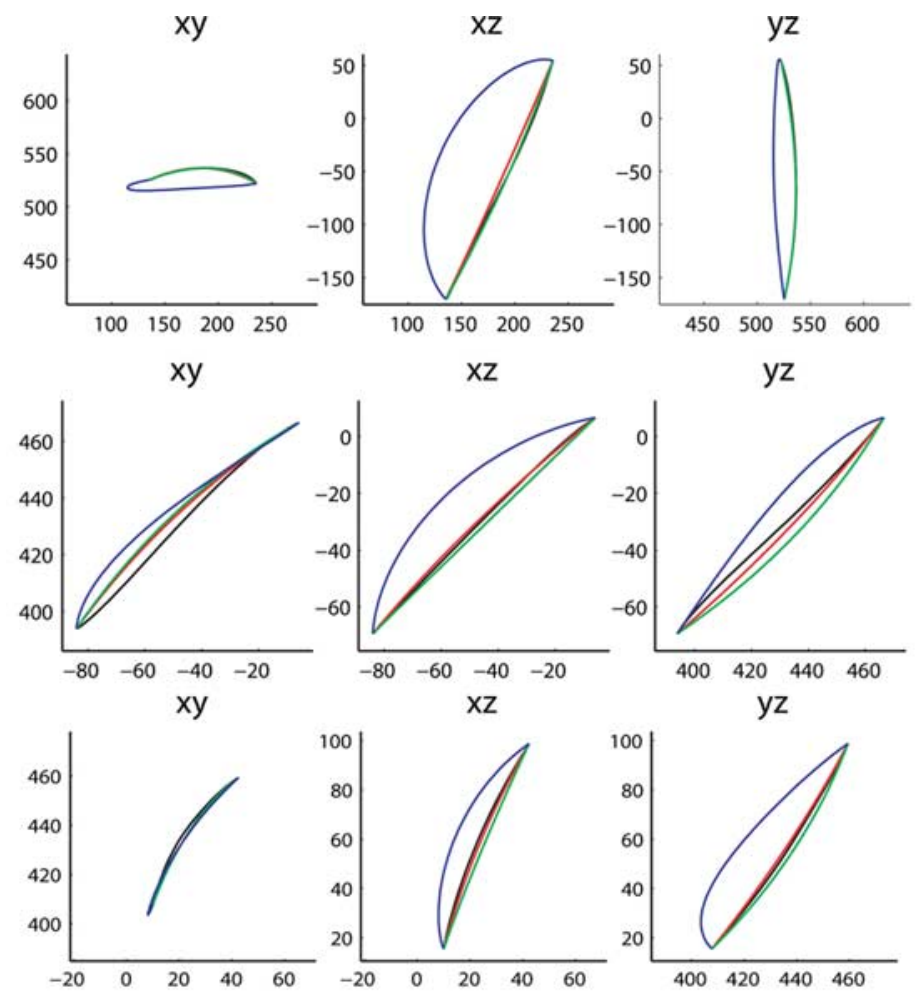

xy
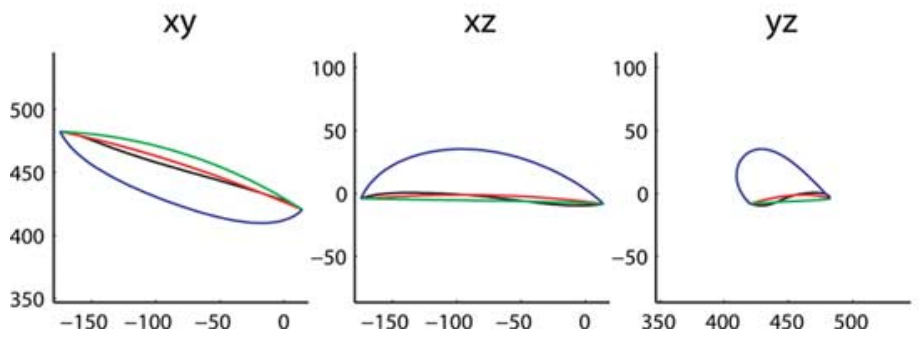

Figure 5. Hand paths. Typical examples of measured and predicted 3D hand paths for different models in the frontoparallel movement condition. Description as in the radial condition.

\section{Temporal level: speed profiles}

We first analyzed the characteristics of the observed tangential hand-speed profiles and investigated whether our modeling assumptions on the temporal level were justified. For this purpose, the normalized speed profiles, $\tilde{v}(\tau)=v(\tau) /\langle v\rangle$, were analyzed, where the average velocity is given by $\langle v\rangle=S / T$ and $\tau=t / T$ defines normalized time. The normalization guarantees that all speed profiles are independent of the distance traveled and satisfy the following relationship: $\int_{0}^{1} \tilde{v}(\tau) d \tau=1$. To assess deviations from the symmetric speed profile, we defined an asymmetry index as the ratio $\left(S_{l}-S_{r}\right) / S$, where $S_{l}$ is the distance traveled up to peak time of velocity (acceleration phase) and $S_{r}$ is the distance traveled from peak time of velocity until the end of the movement (deceleration phase). $S$ denotes the total distance traveled. The temporal evolution of hand paths according to the minimumjerk description assumes that the peak amplitude of the normalized speed profile should invariably reach a value of 1.875 at time $\tau=0.5$. In addition, the bell-shaped minimum-jerk speed profile has an asymmetry index of zero.

The distributions around the mean values (by subjects and movement types) for peak amplitude, peak time, and asymmetry index are given in Figure 9 for radial movements and in Figure 10 for frontoparallel movements. These distributions suggest that the minimum-jerk model provides a reasonable fit to the mean values of amplitude, peak time, and asymmetry factor, although the amplitude for 3D arm movements may be better predicted by the minimum-snap model ( snap $=$ fourth derivative of handposition vector), which would lead to a value of 2.186 instead of 1.875 resulting from the minimum-jerk model (Richardson and Flash, 2002). Measured acceleration times were on average slightly shorter than predicted by the minimum-jerk model, as indicated by the peak times of the hand-speed profiles. The mean asymmetry factor of the speed profiles was small and negative, showing that the distance traveled during the deceleration phase was slightly longer than the distance covered during the acceleration phase. Overall, the MJ model formulated for the arc length of the hand path is a good approximation, although it cannot explain all temporal features of the measured hand movements.

Randomly selected examples of predicted speed profiles resulting from the MJ model along the hand path and the MTC model, superimposed on measured speed profiles, are shown in Figure 11 for the radial movement type and in Figure 12 for movements on the frontal plane. The predicted MJ profiles are in good agreement with the experimental data, although the predicted peak amplitude slightly undershoots the experimental value, and the observed small deviations from the symmetric bell-shaped form cannot be explained by the MJ model. In contrast, the peak amplitudes of the MTC speed profiles are in general too small and show double peaks, in disagreement with the observed data.

The speed profiles were further examined by using a global error measure as in Nakano et al. (1999). The error measure was based on the area that the normalized speed profile encloses with the time axis. When comparing the predicted and measured normalized speed profiles, the compounded area surrounded by the two profiles was divided into a common and a noncommon portion. The ratio of the noncommon area to the whole area defined a speed deviation index (SDI) for each profile (Fig. 6C). The SDI values for the different models are listed in Table 1. Note the large difference for the SDI measures between the GEO and the MTC model and the fact that the SDI values for the GEO and the SJD model are identical.

The results of the mixed-design ANOVAs ( 3 models $\times 2$ conditions of movement) with repeated measures on the last factor were performed using the different error measures as the dependent variables. The results showed that regardless of the error variable used to assess the model-observed differences, the frontal and radial movement conditions did not significantly differ from each other. The interactions shown in Figure 13 did not achieve statistical significance either. However, a major effect of models was found using path, speed, and posture error measures $(p<0.001$ in all cases). Post hoc pairwise comparison showed that the model effects were caused by the larger discrepancies 
A

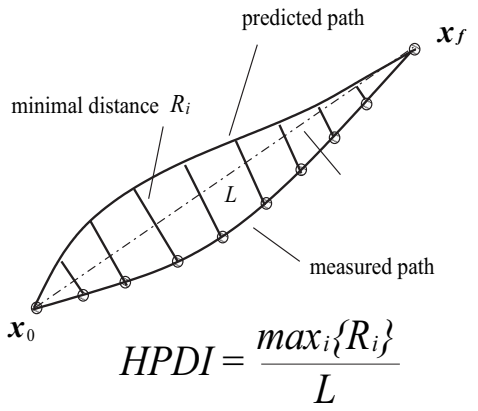

$\mathrm{B}$

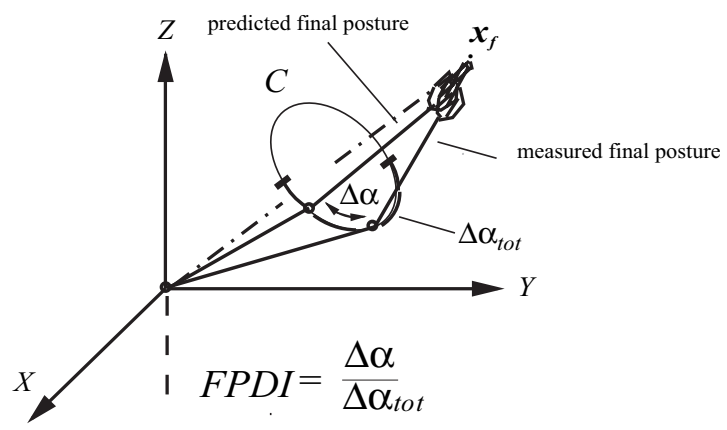

$\mathrm{C}$

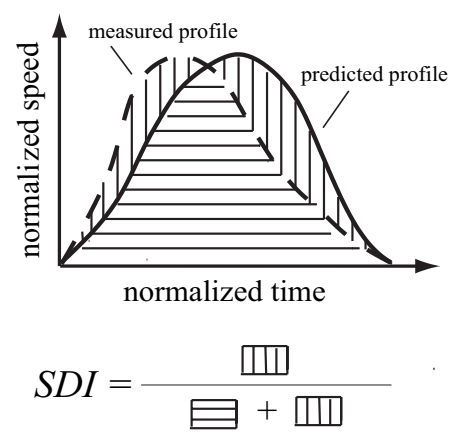

Figure 6. Definition of error measures. $A$, The HPDI of measured and predicted hand paths is the ratio of the maximum of minimal distances, $R=\max _{i=1, \ldots, M-1}\left\{R_{i}\right\}$, between the two paths and the distance, $L$, between the initial and the final target. $B$, At a fixed final hand location $\boldsymbol{x}_{f}$ the elbow can still rotate around an axis going to the shoulder and the final hand location. The measured and the predicted arm posture can thus be defined by the rotation angles, $\alpha_{\text {exp }}$ and $\alpha_{\text {pred }}$, respectively. The FPDI is the ratio of difference in rotation angle around this axis between the measured and predicted posture, $\Delta \alpha=\left|\alpha_{\exp }-\alpha_{\text {pred }}\right|$, and the total anatomical accessible angular range, $\Delta \alpha_{\text {tot }}$ that the arm can sample at the given hand location $\boldsymbol{x}_{f}$. Note that the total angular range depends on the hand location. The FPDI measure thus defines a relative error for the final posture. $\boldsymbol{C}$, The SDI is defined as the ratio of the noncommon area that is enclosed by the speed profiles with the time axis and the total enclosed area.

when the MTC model was assessed regardless of the movement type. The post hoc tests also showed that the MTC model differed significantly $(p<0.001)$ from the GEO and SJD models with respect to the path, speed, and posture error measures. However, the latter two were similar in terms of path error and identical in speed error measures. The GEO and SJD models differed from each other in terms of the posture error (post hoc pairwise comparison shows a borderline value $p=0.0537$ ), with an advantage for the former model, suggesting that the GEO model was the best to predict the posture of the arm. Finally, it is worthwhile mentioning that within the narrow error margins measured for path, speed, and posture, subjects differed from each other. However, all subjects showed the same relative differences with respect to the predictions of the GEO, SDJ, and MTC models. Therefore, the present results suggest that the MTC model is not successful in predicting path, speed, or posture during point-to-point hand movements in 3D. The GEO model proposed in the current study yielded the best results.

\section{Spatiotemporal level: joint-angular positions}

The model presented here assumes that the two preplanned aspects of the movement are integrated at some stage into one spatiotemporal representation. The joint-angular trajectories are an outcome of this integration process and are computed as described at the end of the model-description section.

Figures 14 and 15 show several examples selected at random of predicted and measured joint-angular trajectories for the three shoulder angles and the flexion angle at the elbow joint. Note that the arm configuration at the end point was not specified a priori in the computational model, and thus, the predicted and measured final joint angles may differ. In particular, several examples show large deviations for the humeral angle $\zeta$ in the frontal plane movement conditions. Calculations of the forward kinematics from the predicted joint angles confirm that the large differences in the predicted versus measured humeral rotation are compensated by much smaller differences in other joint angles, such that the discrepancy is indeed restricted to the null space and the boundary condition of reproducing the final hand position has been met. One explanation for this discrepancy might be that this degree of freedom is least controlled by the system. Note that in the case of a fully extended arm, the humeral angle $\zeta$ is identical to the rotation angle $\alpha$ (up to a constant) around the axis going through the shoulder location and the final hand location. Recall that the angle $\alpha$ parameterizes the null space of the end-effector location, and thus, changes in the angle $\alpha$ do not contribute to the task goal.

\section{Dynamic level: driving torques}

The computational model derived at the temporal and geometric levels leads to interesting properties at the dynamic level. These properties will be derived next.

First, we analyze the driving torques needed to generate the movement along the geodesic paths. The dynamic equations of motion for the arm are given by

$$
M(\boldsymbol{q}) \ddot{\boldsymbol{q}}+C(\boldsymbol{q}, \dot{\boldsymbol{q}}) \dot{\boldsymbol{q}}+N(\boldsymbol{q})=\boldsymbol{\tau},
$$

where $N$ denotes the vector of gravitational torques. Frictional torques are neglected. The joint torques, $\tau$, generated by muscle forces are divided into the driving torques, $\tau_{d}$, and the torques, $\tau_{g}$, that counterbalance the external torques generated by the gravitational field. Thus, we assume that gravitation does not contribute to the driving torques along the path in configuration space, but leads to a static posture maintenance at each location in task space. Therefore, we decompose the torques as follows:

$$
\begin{gathered}
\boldsymbol{\tau}=\boldsymbol{\tau}_{d}+\boldsymbol{\tau}_{\mathrm{g}}, \\
\boldsymbol{\tau}_{g}=N(\boldsymbol{q}) .
\end{gathered}
$$

With these assumptions, the dynamic equations (Eq. 48) transform into

$$
M(\boldsymbol{q}) \ddot{\boldsymbol{q}}+C(\boldsymbol{q}, \dot{\boldsymbol{q}}) \dot{\boldsymbol{q}}=\boldsymbol{\tau}_{d} .
$$

As pointed out in previous research works (Flash and Hollerbach, 1982; Atkeson and Hollerbach, 1985), the separation of torques into posture maintenance torques and gravity-independent driving torques might be used by the motor system to simplify arm dynamics. The advantage of such a strategy derives from the scal- 
Table 1. Mean deviation indices ( \pm SD) for hand path, final posture, and hand speed for three models and four subjects in radial and frontal movement conditions

\begin{tabular}{|c|c|c|c|c|c|c|c|c|c|}
\hline \multirow[b]{2}{*}{ Subject } & \multicolumn{3}{|l|}{ HPDI } & \multicolumn{3}{|l|}{ FPDI } & \multicolumn{3}{|l|}{ SDI } \\
\hline & GEO & SJD & MTC & GEO & SID & MTC & GEO & SJD & MTC \\
\hline \multicolumn{10}{|l|}{ Radial } \\
\hline 1 & $0.0675 \pm 0.0355$ & $0.0837 \pm 0.0417$ & $0.2099 \pm 0.0984$ & $0.0548 \pm 0.1481$ & $0.0761 \pm 0.1739$ & $0.1081 \pm 0.2259$ & $0.1175 \pm 0.0902$ & $0.1175 \pm 0.0902$ & $0.3404 \pm 0.0993$ \\
\hline 2 & $0.0765 \pm 0.0450$ & $0.0859 \pm 0.0492$ & $0.1903 \pm 0.1178$ & $0.0619 \pm 0.1732$ & $0.1272 \pm 0.2297$ & $0.1240 \pm 0.2313$ & $0.1557 \pm 0.0995$ & $0.1557 \pm 0.0995$ & $0.3516 \pm 0.1019$ \\
\hline 3 & $0.0943 \pm 0.0629$ & $0.0996 \pm 0.0660$ & $0.2644 \pm 0.1219$ & $0.0518 \pm 0.1404$ & $0.0545 \pm 0.1468$ & $0.1637 \pm 0.2857$ & $0.1299 \pm 0.0861$ & $0.1299 \pm 0.0861$ & $0.4533 \pm 0.0917$ \\
\hline 4 & $0.0961 \pm 0.0655$ & $0.0970 \pm 0.0657$ & $0.2930 \pm 0.1102$ & $0.0570 \pm 0.1643$ & $0.0797 \pm 0.1906$ & $0.1498 \pm 0.2696$ & $0.1397 \pm 0.0949$ & $0.1397 \pm 0.0949$ & $0.4187 \pm 0.0985$ \\
\hline \multicolumn{10}{|l|}{ Frontal } \\
\hline 1 & $0.0770 \pm 0.0442$ & $0.0878 \pm 0.0476$ & $0.2586 \pm 0.0853$ & $0.0346 \pm 0.1279$ & $0.0675 \pm 0.1547$ & $0.1102 \pm 0.2129$ & $0.1014 \pm 0.0813$ & $0.1014 \pm 0.0813$ & $0.3490 \pm 0.1240$ \\
\hline 2 & $0.0748 \pm 0.0551$ & $0.0811 \pm 0.0539$ & $0.2454 \pm 0.0858$ & $0.0628 \pm 0.1621$ & $0.1685 \pm 0.2473$ & $0.1954 \pm 0.2712$ & $0.1742 \pm 0.1059$ & $0.1742 \pm 0.1059$ & $0.3561 \pm 0.1062$ \\
\hline 3 & $0.0927 \pm 0.0618$ & $0.0977 \pm 0.0644$ & $0.3781 \pm 0.1009$ & $0.0604 \pm 0.1337$ & $0.0817 \pm 0.1526$ & $0.1591 \pm 0.2691$ & $0.1622 \pm 0.1080$ & $0.1622 \pm 0.1080$ & $0.4193 \pm 0.0958$ \\
\hline 4 & $0.0955 \pm 0.0389$ & $0.1053 \pm 0.0341$ & $0.3536 \pm 0.0796$ & $0.0315 \pm 0.1175$ & $0.0344 \pm 0.0920$ & $0.1022 \pm 0.2285$ & $0.1799 \pm 0.1139$ & $0.1799 \pm 0.1139$ & $0.4590 \pm 0.1134$ \\
\hline
\end{tabular}

elevation

azimuth
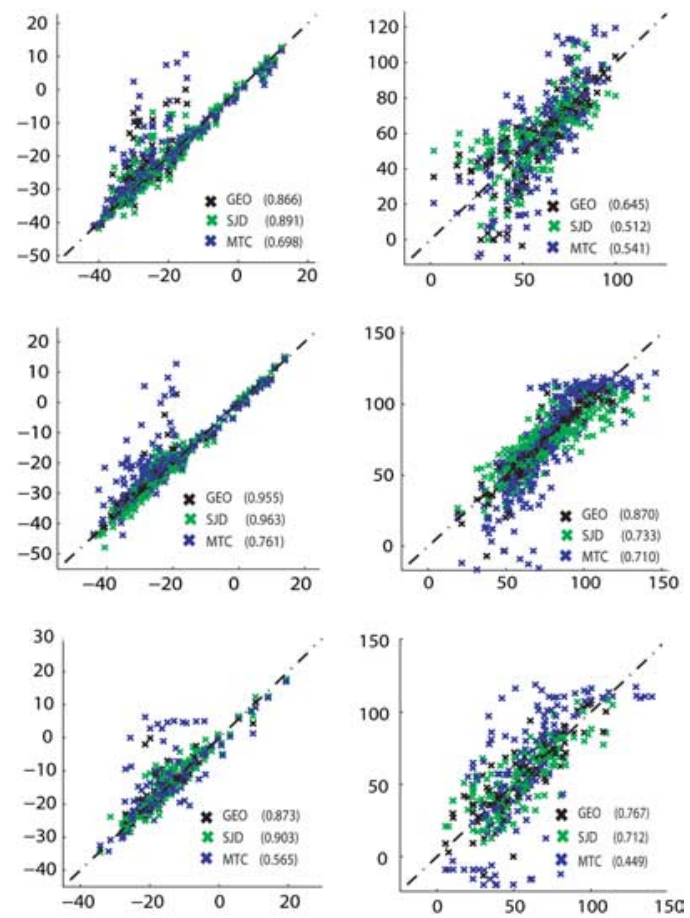
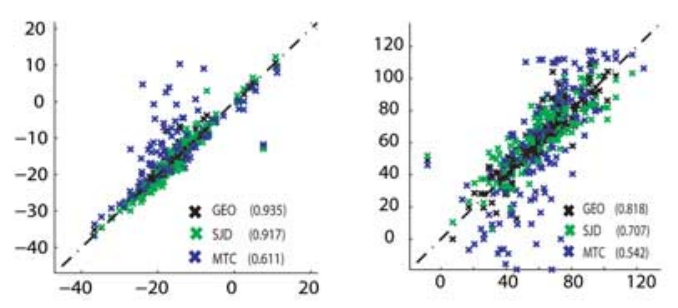

measured angle (deg)

S4

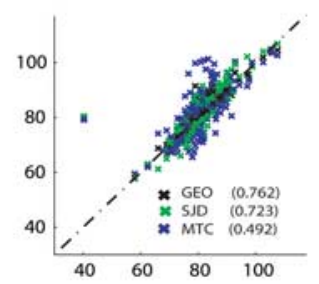

)

Figure 7. Final arm postures. Comparison of the model predictions for the elevation, azimuth, and torsion angles with the experimental data in the radial movement condition for all four subjects $51-S 4$. The $R^{2}$ values for the three computational models (GEO, SJD, and MTC) are given as insets in the figures. The GEO model led to the highest score in $R^{2}$ values followed by the SJD and MTC models.

ing properties of the driving torques under a change of speed. Consider a trajectory that results from a given trajectory by time scaling $\tilde{t}=k t$ with some constant $k$.

Then, hand speed scales according to

$$
\tilde{v}=\frac{d \tilde{s}(\tilde{t})}{d \tilde{t}}=\frac{d s(t(\tilde{t}))}{d \tilde{t}}=\frac{d s(t)}{d t} \frac{d t}{d \tilde{t}}=\frac{1}{k} v .
$$

For $k<1$, the movement speed is increased, whereas for $k>1$, the movement is slowed down. The driving torques scale according to Equation 51 as

$$
\tilde{\boldsymbol{\tau}}_{d}=\frac{1}{k^{2}} \boldsymbol{\tau}_{d}
$$

For example, to move the hand twice as fast along a given hand path, the driving torques have to be multiplied by a factor of four.

We further analyze the driving torques by assuming that the path in configuration space is a priori specified and the jointangular trajectory can be represented in the form $\boldsymbol{q}(t)=\boldsymbol{q}(\lambda(t))$, where $\lambda=\sigma / \Sigma$ is the normalized arc length in configuration space. Using the chain rule, the equations of motion in Equation 51 can be written as

$$
\begin{array}{r}
M(\boldsymbol{q}) \boldsymbol{q}^{\prime} \ddot{\lambda}+\left[M(\boldsymbol{q}) \boldsymbol{q}^{\prime \prime}+C\left(\boldsymbol{q}, \boldsymbol{q}^{\prime}\right) \boldsymbol{q}^{\prime}\right] \dot{\lambda}^{2} \\
=\boldsymbol{\tau}_{d},
\end{array}
$$

where a prime and a dot denote differentiation with respect to $\lambda$ and time, respectively.

For movements along geodesic paths, the term in brackets on the left side vanishes according to Equation 31. The vanishing expression consists of inertial torques that depend on the speed in configuration space, $\boldsymbol{\tau}_{2}=M(\boldsymbol{q}) \boldsymbol{q}^{\prime \prime} \dot{\lambda}^{2}$, and the torques that are commonly denoted as the centrifugal and Coriolis torques (Flash and Hollerbach, 1982), $\boldsymbol{\tau}_{3}=C\left(\boldsymbol{q}, \boldsymbol{q}^{\prime}\right) \boldsymbol{q}^{\prime} \dot{\lambda}^{2}$ $=C(\boldsymbol{q}, \dot{\boldsymbol{q}}) \dot{\boldsymbol{q}}$; i.e., along geodesics, it is $\boldsymbol{\tau}_{2}+$ $\boldsymbol{\tau}_{3}=0$. The remaining term $\boldsymbol{\tau}_{1}=M(\boldsymbol{q}) \boldsymbol{q}^{\prime} \ddot{\lambda}$ describes inertial torques that depend linearly on the acceleration in configuration space. Thus, the arm dynamics for movements along geodesic paths with nonconstant speed is governed by

$$
\boldsymbol{\tau}_{d}=\boldsymbol{\tau}_{1}=M(\boldsymbol{q}) \boldsymbol{q}^{\prime} \ddot{\lambda} .
$$

Note that the movement along geodesic paths results in a much simpler dynamics than given in Equation 48. We remark further that the Coriolis and centrifugal interaction torques, $\boldsymbol{\tau}_{3}$, can still 
be present for arm movements along geodesic paths as long as they compensate the speed-dependent inertial torques $\boldsymbol{\tau}_{2}$.

To analyze further the dynamical properties of movements along a geodesic path, we compare Equation 54 to Newton's equation of motion for a mass point $m$ along a prespecified path, $\boldsymbol{x}(s)$, where $s$ is the Euclidean arc length along the path. Newton's equation of motion, $\boldsymbol{F}=m \ddot{\boldsymbol{x}}$, transforms with $\dot{\boldsymbol{x}}=s \dot{\boldsymbol{x}}^{\prime}$ and $\ddot{\boldsymbol{x}}=\ddot{\boldsymbol{s}} \boldsymbol{x}^{\prime}+\dot{s}^{2} \boldsymbol{x}^{\prime \prime}$ to

$$
\ddot{s} \boldsymbol{t}+\boldsymbol{\kappa} \dot{s}^{2} \boldsymbol{n}=\boldsymbol{F} / m,
$$

where a dot and a prime denotes differentiation with respect to time and arc length $s$, respectively. The vectors $\boldsymbol{t}(s)=\boldsymbol{x}^{\prime}(s)$ and $\boldsymbol{n}(s)=\boldsymbol{x}^{\prime \prime}(s) / \kappa(s)$ are the tangential and normal vector to the path, respectively, and $\kappa(s)$ denotes the curvature of the path. Note the similar structure of Equations 54 and 56. If the mass point moves on a straight path (geodesics in Euclidean space) where $x^{\prime \prime}=0$, accelerations act according to Equation 56 only in directions tangential to the path, whereas accelerations normal to the path disappear.

Similar results hold for movements of the arm according to Equation 54. For further analysis, it is useful to multiply Equation 54 with the inverse of the metric, leading to

$$
\begin{aligned}
\boldsymbol{q}^{\prime} \ddot{\lambda}+M^{-1}(\boldsymbol{q})\left[M(\boldsymbol{q}) \boldsymbol{q}^{\prime \prime}+\right. \\
\left.C\left(\boldsymbol{q}, \boldsymbol{q}^{\prime}\right) \boldsymbol{q}^{\prime}\right] \dot{\lambda}^{2}=M^{-1}(\boldsymbol{q}) \boldsymbol{\tau}_{d} .
\end{aligned}
$$

For arm movements along geodesic paths ("straight" paths in Riemannian configuration space), the expression in the square brackets of Equation 57 disappears, and thus all accelerations act in direction tangential to the path (i.e., along $\boldsymbol{q}^{\prime}$ ), whereas all nontangential accelerations vanish. Movements along geodesics thus require less muscular effort, because only forces that induce acceleration in direction tangential to the path in configuration space have to be provided by the muscles. Any deviation from the straight (geodesic) path requires additional muscle forces. These forces are needed to keep the arm on the nongeodesic path ("curved" path in Riemannian configuration space) comparable with the centripetal force needed to keep a mass point on a (curved) circular path in Euclidean space.

Several examples of predicted and measured driving torques in the radial and frontoparallel movement conditions are shown in rows in Figures 16 and 17, respectively. The measured driving torques were obtained by evaluating the left side of Equation 51 using the experimental data. The predicted driving torques were derived from Equation 55 using the prediction of the GEO model. The first column in Figures 16 and 17 shows the total driving torque. The middle column shows the inertial torque $\boldsymbol{\tau}_{1}$, and the last column depicts the sum of all configuration-speeddependent torques, $\boldsymbol{\tau}_{2}+\boldsymbol{\tau}_{3}$. Note that according to the GEO model, $\boldsymbol{\tau}_{2}+\boldsymbol{\tau}_{3}=0$, and thus the inertial torque $\boldsymbol{\tau}_{1}$ is equal to the elevation

azimuth

torsion
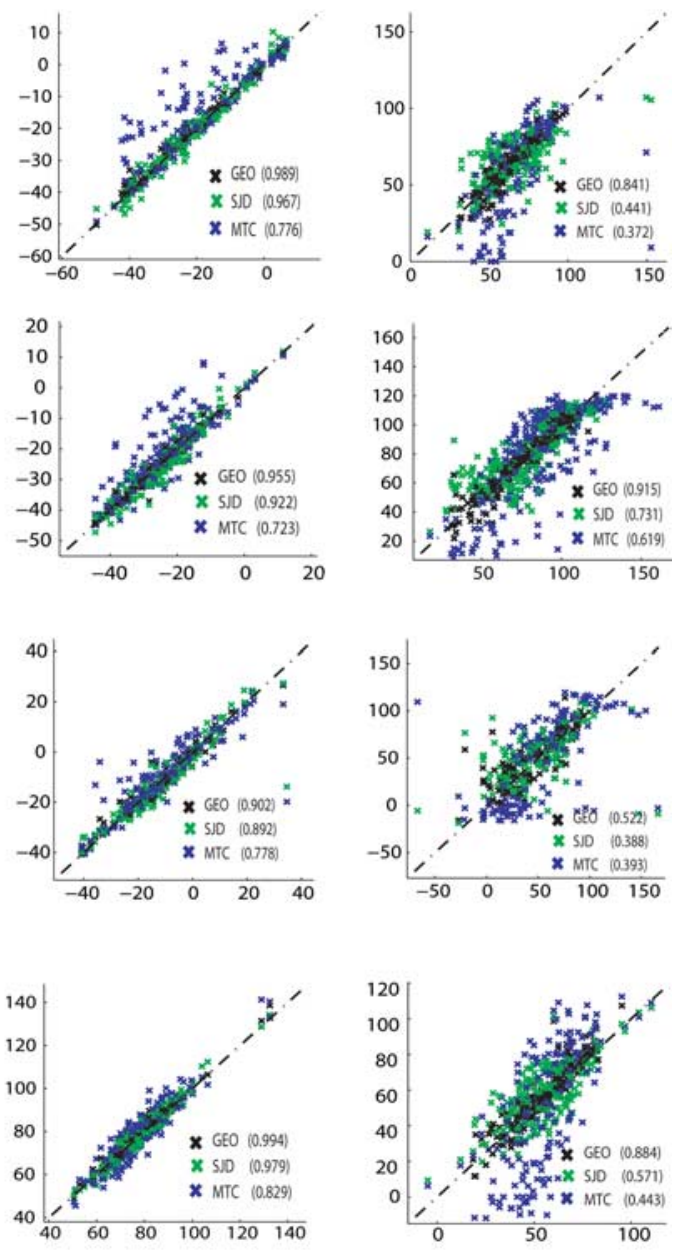

measured angle (deg)

Figure 8. Final arm postures. Comparison of the model predictions for the elevation, azimuth, and torsion angles with the experimental data in the frontoparallel movement condition for all four subjects S1-S4. Description as in the radial condition.

total driving torque $\boldsymbol{\tau}_{d}$. The measured torque profiles resulted in larger variability around the predicted curves. It is worth noting that the sign and size of the torque amplitudes were well accounted for by the model but the measured torque profiles showed larger fluctuations. We can think of several reasons for this discrepancy. First, the path does not always follow a geodesic path, and thus, Equation 55 does not hold exactly. Second, observed speed profiles show deviations from a symmetric profile, and thus, the assumption of MJ speed profile along the hand path is too stringent and cannot account for the small fluctuations in the torque profiles. Finally, the extraction of torques from position measurements is not trivial, and noise may be induced during data processing. Also note that the sum of measured torques, $\boldsymbol{\tau}_{2}+\boldsymbol{\tau}_{3}$, nearly vanishes in the presented examples, which suggests that most of the driving torques can be attributed to the torques $\boldsymbol{\tau}_{1}$. These results are in good agreement with the GEO model.

The implication of Equation 55 in task space can be derived by introducing the operational force $\boldsymbol{F}$ at the end effector and by reparameterization with respect to arc length, $s$, of the Euclidean 
normalized speed peak amplitude peak time asymmetry curvature index
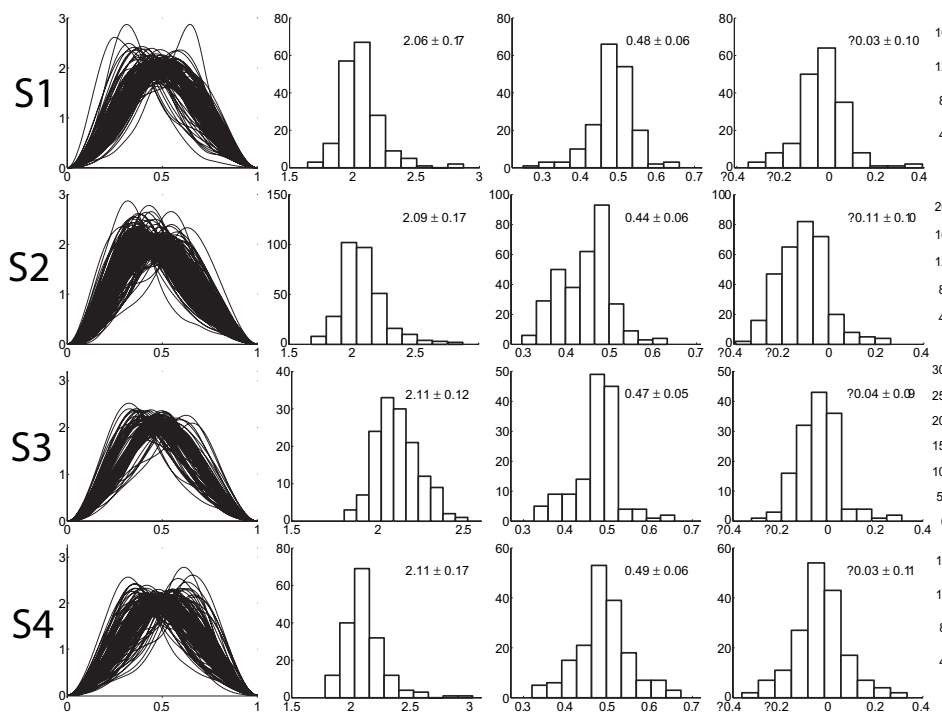

Figure 9. Descriptive hand-speed statistics and curvature index. Normalized speed profiles for the four subjects S1-S4 in the radial movement condition. The distributions of peak amplitude, peak time, asymmetry factor, and curvature index are shown in the subplots. The mean peak amplitude is always larger than that predicted by the MJ model (1.875), and peak time occurs slightly before the prediction of the MJ model (0.5). The deviation of the mean asymmetry index from the MJ model $(0)$ is small. Overall, the MJ model formulated for the arc length of the hand path is a good model assumption. Most of the measured movements resulted in quasi-straight hand paths with a curvature index $\mathrm{Cl} \leq 1.05$.

normalized speed peak amplitude peak time

asymmetry curvature index
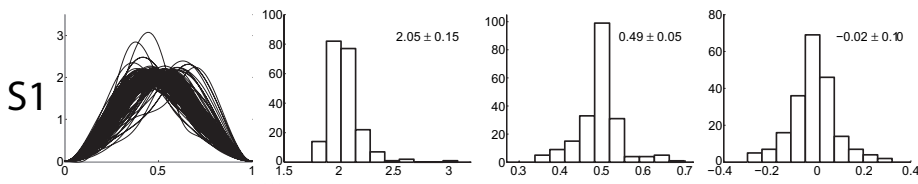

$\mathrm{S} 2$
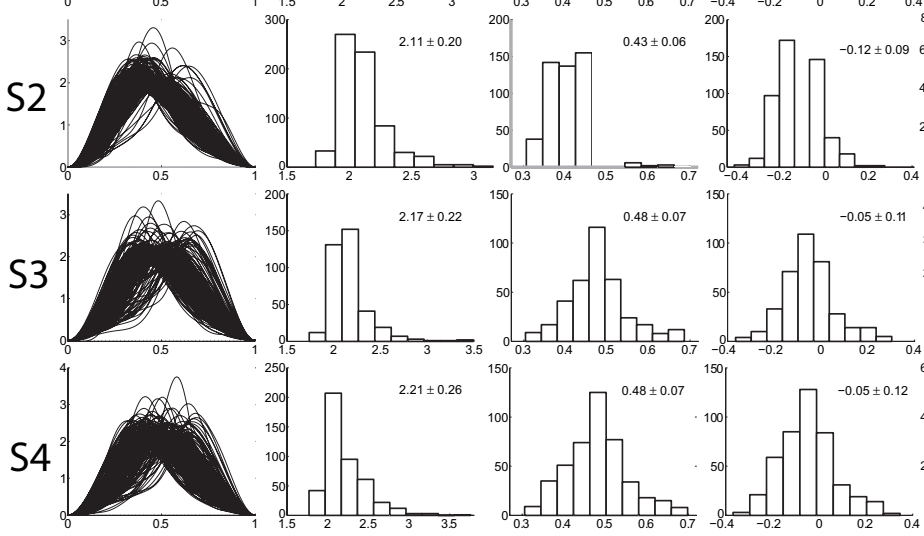

Figure 10. Descriptive hand-speed statistics and curvature index. Normalized speed profiles for the four subjects S1-S4 in the frontoparallel movement condition. Description as in the radial condition.

hand path. The driving torques $\boldsymbol{\tau}_{d}$ are related to the operational force $\boldsymbol{F}$ by the following fundamental relation (Kathib, 1987):

$$
\boldsymbol{\tau}_{d}=J_{h}^{T} \boldsymbol{F}
$$

Inserting Equation 58 into Equation 57 and multiplying both sides with the hand Jacobian leads for geodesic paths to

$$
\ddot{\lambda} \boldsymbol{x}^{\prime}=J_{h} M^{-1} J_{h}^{T} \boldsymbol{F}=W F,
$$
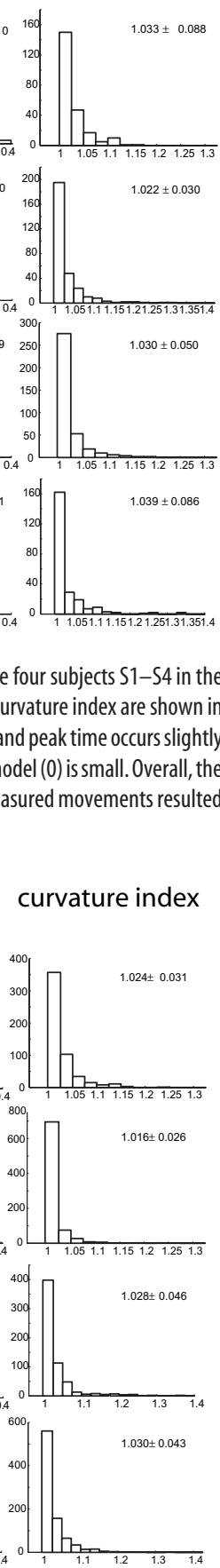

where we have used the relation $\boldsymbol{x}^{\prime}=J_{h} \boldsymbol{q}^{\prime}$, the definition of the mobility matrix $W=$ $J_{h} M^{-1} J_{h}^{T}$, and the fact that the expression in square brackets in Equation 57 disappears along geodesic paths.

Next, we reparameterize Equation 59 with respect to Euclidean arc length $s$ by inserting the function $\lambda=\lambda(s)$ and Equation 44 into Equation 59. With $d x / d \lambda=$ $(d x / d s)(d s / d \lambda)=(d x / d s)\left(1 /\left[\lambda^{\prime}(s)\right]\right)$, we get

$$
\left[\ddot{s}+\frac{\lambda^{\prime \prime}(s)}{\lambda^{\prime}(s)} \dot{s}^{2}\right] \boldsymbol{t}(\mathrm{s})=W(\boldsymbol{q}(s)) \boldsymbol{F}(s),
$$

where $\boldsymbol{t}=d \boldsymbol{x} / d s$ is the unit tangent vector to the hand path and a prime now denotes differentiation with respect to $s$.

The second term in the square brackets of Equation 60 depends on the curvature of the hand path. For quasi-straight hand paths, however, the term can be neglected, because for such paths, $\lambda^{\prime \prime}(s) \approx 0$; i.e.,

$$
a_{t}=\ddot{s} \boldsymbol{t}=W \boldsymbol{F},
$$

where $\boldsymbol{a}_{t}$ is the tangential acceleration.

Note also that for quasi-straight hand paths, the driving torques along geodesic paths in configuration space are according to Equation 55 well approximated by

$$
\begin{array}{r}
\boldsymbol{\tau}_{d}=M(\boldsymbol{q}(s)) \boldsymbol{q}^{\prime}(s)\left[\ddot{s}+\frac{\lambda^{\prime \prime}(s)}{\lambda^{\prime}(s)} \dot{s}^{2}\right] \approx \\
M(\boldsymbol{q}(s)) \boldsymbol{q}^{\prime}(s) \ddot{s},
\end{array}
$$

resulting in a linear relation between the hand acceleration $\ddot{s}$ and the driving torques. The predictions of the GEO model are compatible with the assumption of quasi-straight hand paths as shown in Figures 4 and 5 as well as the experimental measured hand paths that lead according to Figures 9 and 10 to quasi-straight hand-path distributions of the curvature index.

We conclude from Equation 61 that the tangential acceleration in task space is identical to the product of the mobility matrix, which measures the ease of accelerating the hand in a certain direction, and the actual force. Conversely, for movements along quasi-straight hand paths that were derived from geodesic paths in configuration space, the product of mobility matrix and operational force (effective acceleration) is always pointing in direction tangential to the hand path. The total acceleration of the end effector follows then as $\boldsymbol{a}=\boldsymbol{a}_{t}+\boldsymbol{a}_{n}$, where $\boldsymbol{a}_{n}$ is the normal acceleration given by $\boldsymbol{a}_{n}=\boldsymbol{\kappa} \dot{\boldsymbol{s}}^{2} \boldsymbol{n}$. The vector $\boldsymbol{n}$ denotes the normal vector and $\kappa$ the curvature of the hand path. It should be noted that the tangential and normal vectors as well as the 
curvature of the hand path are known quantities that follow from the projection of the geodesic path into task space.

We will next analyze what the selected temporal feature (speed) of the computational model implies at the dynamical level. We first consider the minimum torque-change model (Uno et al., 1989; Nakano et al., 1999; Wada et al., 2001). We analyze what this criterion would imply assuming that the movement is along a geodesic path and the speed along the hand path, $\dot{s}(t)$, is the only free variable to be determined. Along a geodesic path, the cost associated with the minimum torquechange model is

$C=$

$$
\begin{gathered}
\int_{0}^{T} \dot{\boldsymbol{\tau}}_{d}^{2} d t=\int_{0}^{\mathrm{T}}\left(\frac{d}{d t}\left[M(\boldsymbol{q}(s)) \boldsymbol{q}^{\prime}(s) \ddot{s}\right]\right)^{2} d t \\
=\int_{0}^{\mathrm{T}}\left(\frac{d}{d t}[\boldsymbol{A}(t) \ddot{s}]\right)^{2} d t \rightarrow \min , \quad
\end{gathered}
$$

where we have used Equation 62 and defined $\boldsymbol{A}(t) \equiv M(\boldsymbol{q}(s(t))) \boldsymbol{q}^{\prime}(s(t))$. We noticed that for geodesic paths, $\dot{A}(t) \approx 0$, and thus the change of driving torque is to a first approximation proportional to the jerk of the arc length:

$$
C \approx|\boldsymbol{A}|^{2} \int_{0}^{T} \dddot{s}^{2} d t \rightarrow \min
$$

We conclude that the minimization of the squared driving torque change integrated over the movement is roughly equal to a minimization of the squared jerk of the arc length of the hand path integrated over the movement time. The minimum-jerk model and the minimum torque-change model are thus compatible when restricted to geodesic paths in configuration space. Moreover, as shown in Appendix D, the chosen motor patterns at the geometric and temporal levels are equivalent to a minimization of the peak value of the kinetic energy. Thus, the minimum-peak kinetic energy model as suggested by Soechting et al. (1995) is an additional outcome of our model.

A second, alternative solution for the speed determination along the geodesic path may consist of a cost in task space in the form of the squared change in effective acceleration integrated over the movement time, thus:

$$
C=\int_{0}^{T}\left[\frac{d}{d t}(\mathrm{WF})\right]^{2} d t \rightarrow \min
$$

S4
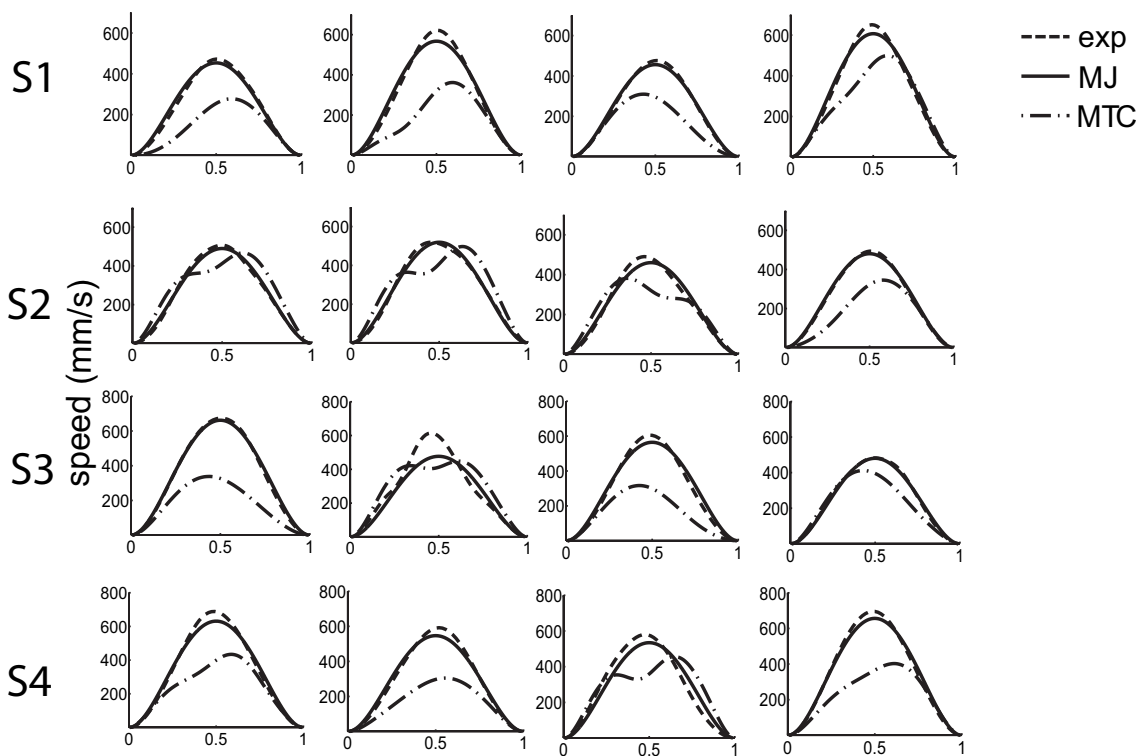

\section{normalized time}

Figure 11. Hand speeds. Typical examples of measured and predicted speed profiles as derived from the MJ and MTC model for the four subjects $\mathrm{S1-S4}$ in the radial movement condition. The peak amplitudes of the MTC model are too small, and in several cases, this model shows double-peaked speed profiles. The MJ model leads to peak amplitudes with small overshoots. Note that the observed small deviations from the symmetric speed profile cannot be accounted for by the MJ model.
S1
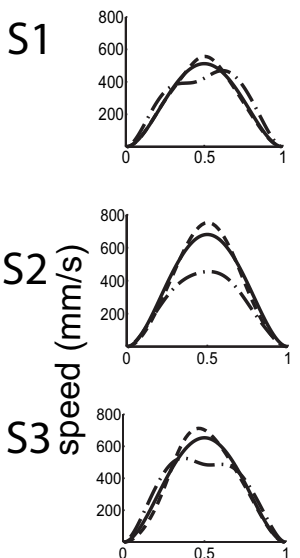

S4

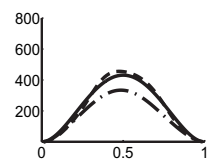

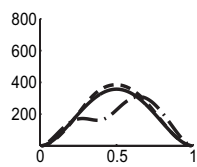
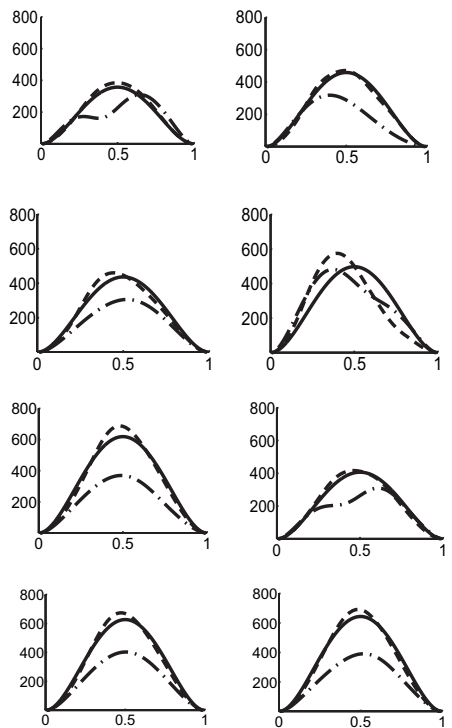

\section{normalized time}
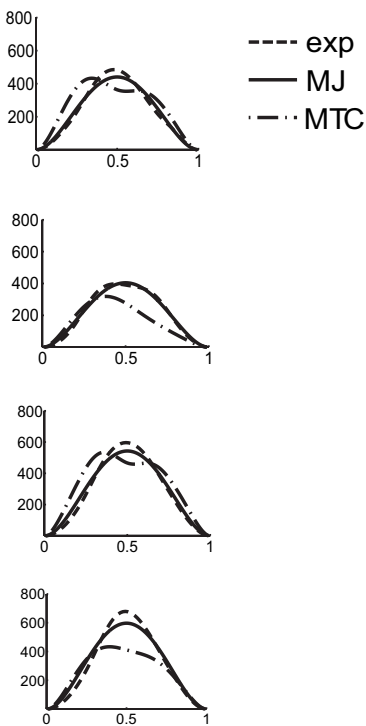

Figure 12. Hand speeds. Typical examples of measured and predicted speed profiles as derived from the MJ and MTC models for the four subjects $\$ 1-\$ 4$ in the frontoparallel movement condition. Description as in the radial condition.
The cost can be rewritten according to Equation 61 as

$$
C=\int_{0}^{T} \dot{\boldsymbol{a}}_{t}^{2} d t=\int_{0}^{T}\left[\frac{d}{d t}(\ddot{s} \boldsymbol{t}(s))\right]^{2} d t=\int_{0}^{T}\left[\ddot{s}^{2}\right.
$$

$$
\left.+(\kappa(s) \dddot{s} \dot{s})^{2}\right] d t \rightarrow \min ,
$$


path

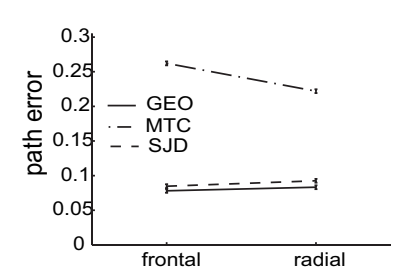

posture

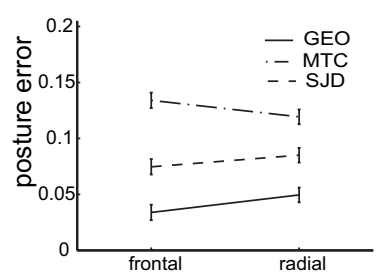

speed

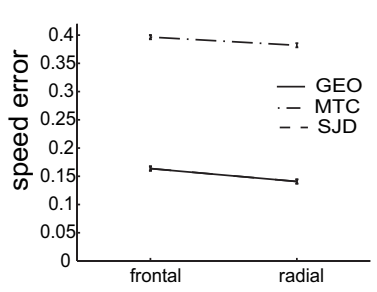

Figure 13. Interactions between models (SDJ, MTC, and GE0) and movement conditions (radial and frontal) using path, speed, and posture error measures as the dependent variables in multiple ANOVAs. Note that the GEO and the SJD models both assume an MJ speed profile, resulting in an identical SDI error measure.

S1
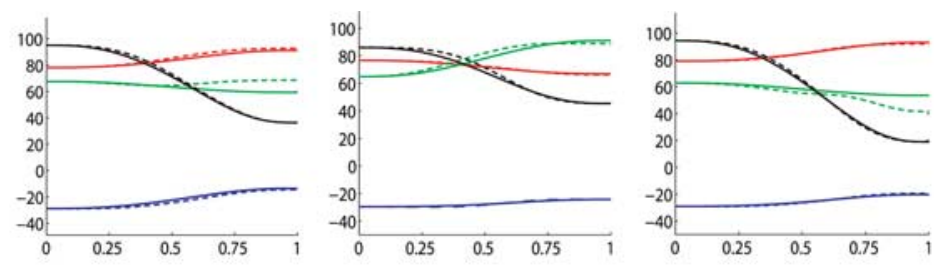

S2
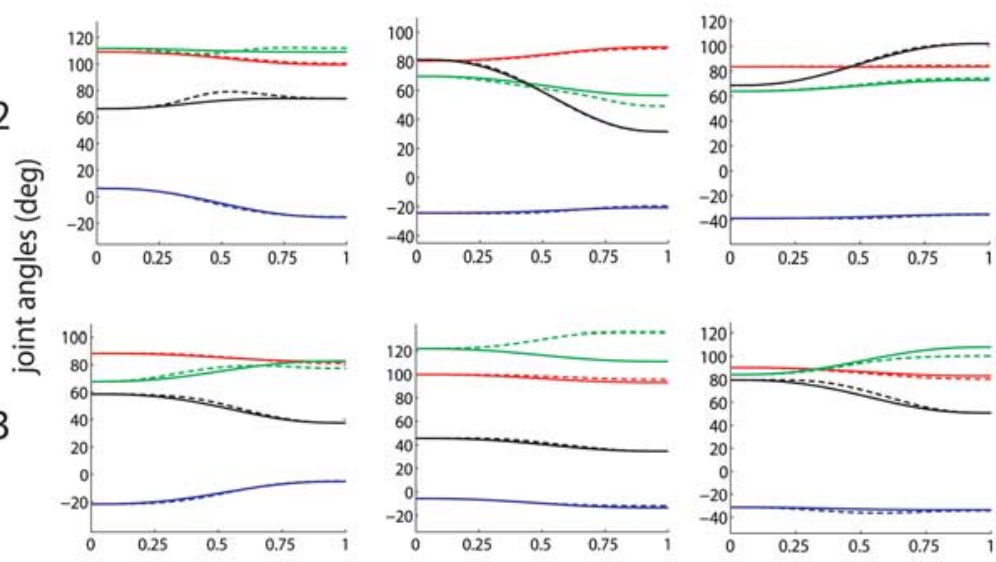

S4

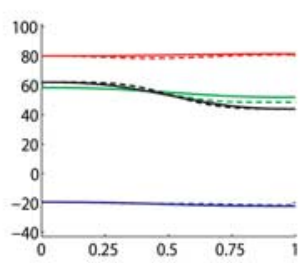

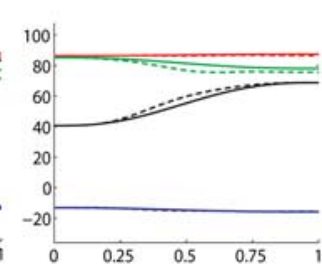

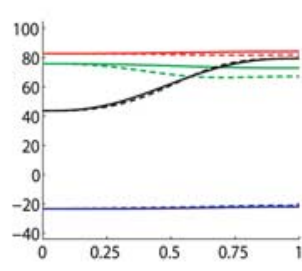

normalized time

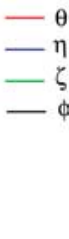

$-\theta$
$-\zeta$

At the dynamic level, we have shown that geodesic paths in configuration space correspond to arm movements with less muscular effort, because only forces that induce acceleration in direction tangential to the geodesic path have to be provided by the muscles. For any other (nongeodesic) path, additional muscle forces have to be provided to generate the configuration-speeddependent interaction torques. Movements along geodesic paths with quasistraight hand paths require driving torques that are linearly related to the tangential acceleration. It follows that the arm dynamic equations along geodesic paths are significantly simplified.

Moreover, we have shown that geodesic paths together with a minimum-jerk speed profile imposed along the extrinsic hand path corresponded to jointangular trajectories with minimal peak kinetic energy over all final accessible arm postures at the target location. Our model is thus compatible with the minimum peak-work model suggested by Soechting et al. (1995), and predicts not only the final arm posture as presented by that model, but also the entire hand path and the joint-angular trajectories.

Our study also provides a dynamic interpretation of the minimum-jerk model by showing that a maximal smoothness criterion imposed on the hand-speed profile leads to a near miniwhere we used the relationships $\boldsymbol{t}^{\prime}(s)=\boldsymbol{\kappa}(s) \boldsymbol{n}(s)$ and $\boldsymbol{t}(s) \cdot \boldsymbol{n}(s)=$ 0 . Because for quasi-straight hand paths, $\kappa(s) \ll 1$, the second term in the last expression of Equation 66 can be neglected, and the cost transforms into the modified minimum-jerk model that defined the temporal properties of the movement in the GEO model.

\section{Discussion}

In this study, we derived a computational model for 3D point-topoint movements that reconciles different existing models into one computational framework. The observed invariance of path

mization of the squared change of driving torques along the selected geodesic path. The observed double-peaked torque profiles may be the result of the simplified dynamics along geodesics. Two prominent and apparently contradictory models, the minimum-jerk model (Flash and Hogan, 1985) and the minimum torque-change model (Uno et al., 1989; Nakano et al., 1999; Wada et al., 2001), could thus be reconciled within the framework of geodesics.

We do not propose that the CNS has an internal representation of Riemannian geometry or geodesics. We hypothesized that geodesics are an emerging property of the system that seeks to 
reduce muscular efforts possibly based on proprioceptive feedback. Along geodesic paths, the sum of configuration-speeddependent inertial torques and centrifugal and Coriolis torques disappears, and only inertial torques that depend on the acceleration in configuration space remain. It is important to note that centrifugal or Coriolis interaction torques along geodesic paths are still possible provided that the net torques, i.e., the sum of the torques $\tau_{2}$ and $\boldsymbol{\tau}_{3}$, disappear. These findings are interesting in light of evidence suggesting that during multijoint movements, muscle activation and neural control strategies have evolved to compensate for interaction torques (Flash and Hollerbach, 1982; Sainburg et al., 1993; Gribble and Ostry, 1999). An equilibrium trajectory control strategy (Feldman, 1966, 1986; Flash, 1987) may enable movement by continuously shifting the arm's equilibrium point along the predefined geodesic path.

\section{Validity of the proposed model}

The predictions of the model for jointangular paths, final posture, hand paths, and speed profiles were in good agreement with our experimental data.

Among the three tested models, the geodesic model accounted best for the observed hand paths and final arm postures. This seems to support previous results demonstrating the role of inertial properties in the selection of the final arm posture (Soechting et al., 1995; Flanders et al., 2003) and the independence of final posture from movement speed and gravity (Nishikawa et al., 1999). In contrast, the MTC model resulted in strongly curved hand paths and unrealistic arm postures. The overall fit of the minimum-jerk speed profile was very good, although observed speed profiles frequently showed slightly larger amplitudes and deviations from the symmetric shape. The speed profiles computed from the MTC model resulted in too-small amplitudes that showed in several cases unrealistic double peaks. The sign and size of torque amplitudes were well accounted for by the model, but all the fine details of the driving-torque profiles could not be explained with the present model.

Simulation studies showed that the application of the geodesic model to planar movements resulted in strongly curved hand paths. This poses the question of whether there is a fundamental difference in the planning and control of movements in $2 \mathrm{D}$ versus $3 \mathrm{D}$ space. One possible explanation for this discrepancy may lie in the fact that planning of movements constrained to a horizontal plane is more strongly influenced by visual feedback and by the wish to move along straight lines as opposed to unconstrained $3 \mathrm{D}$ point-to-point movements and movements in the vertical plane (Atkeson and Hollerbach, 1985; Desmurget et al., 1997).

\section{Evidence for an independent control of temporal and spatial aspects of movement in CNS}

Neurophysiological evidence suggests that spatial aspects of hand paths and their evolution over time may be planned at
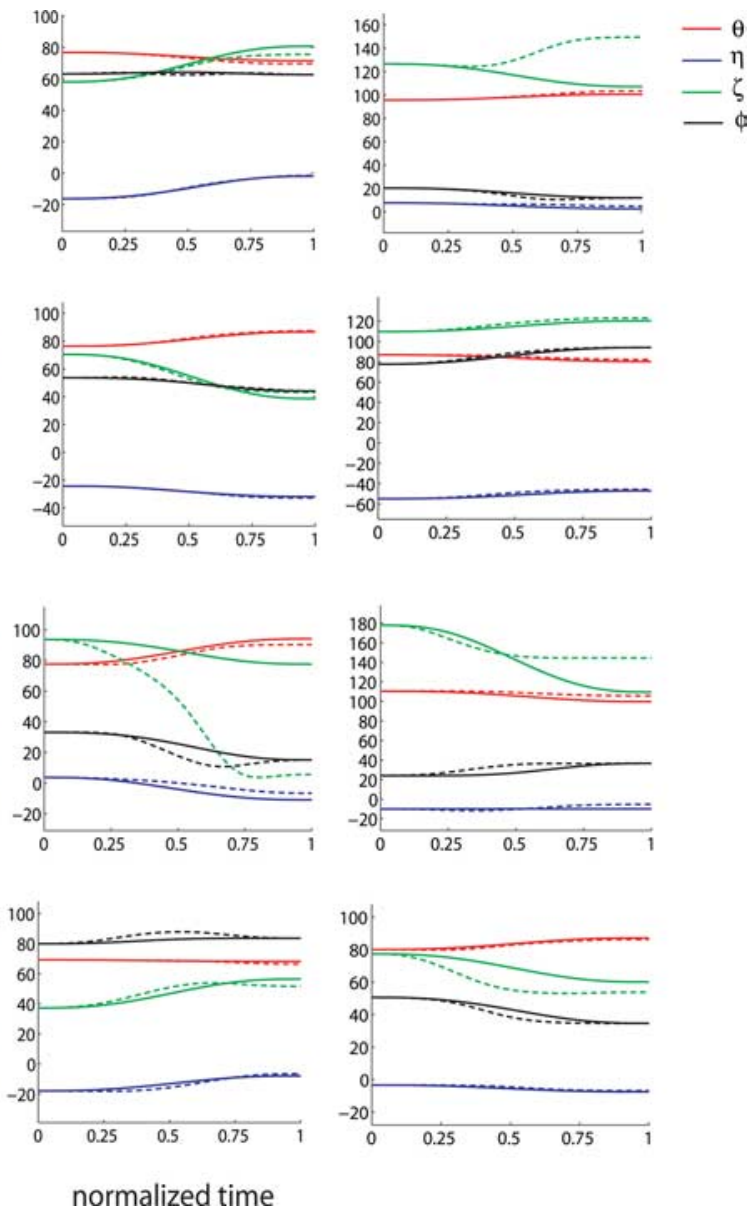

Figure 15. Joint-angular trajectories. Typical examples of predicted (solid) joint-angular trajectories resulting from the integration of the optimal geodesic path in configuration space and the minimum-jerk speed profile along the hand path in task space in the frontoparallel movement condition for all four subjects S1-S4. Description as in the radial condition.

different independent levels. For example, movement direction toward visual targets (Georgopoulos et al., 1988) and changes in movement direction (Georgopoulos et al., 1989) are encoded by directionally tuned cells within the primary motor cortex (Kettner et al., 1988), suggesting that the motor cortex may be one of the specific sites for processing spatial information before the actual movement execution (Georgopoulos, 1995; Scott, 2000).

More complex spatial information related to movement (e.g., during drawing geometrical patterns) seems to be mediated via neural activity in prefrontal and premotor regions (Moran and Schwartz, 1999; Averbeck et al., 2002). In a recent study (Torres and Andersen, 2006), the space-time separation has been shown during the learning of an obstacle-avoidance task in monkeys. It has been proposed that the geometric planning stage is performed by the posterior parietal cortex.

On the other hand, timing is a fundamental feature of motor skill that seems to be widely represented in the CNS (Rao et al., 1997). Previous neurophysiological and brain mapping studies have described a highly distributed network of cortical and subcortical areas that code temporal aspects of the movements (Harrington et al., 1998; Bengtsson et al., 2005; Buhusi and Meck, 2005; Xu et al., 2006). These subcortical areas may include both the cerebellum and the basal ganglia. For example, for simple movements, temporal information is encoded in the anterior and posterior regions of the cerebellar cortex 
$\tau_{\mathrm{d}}$
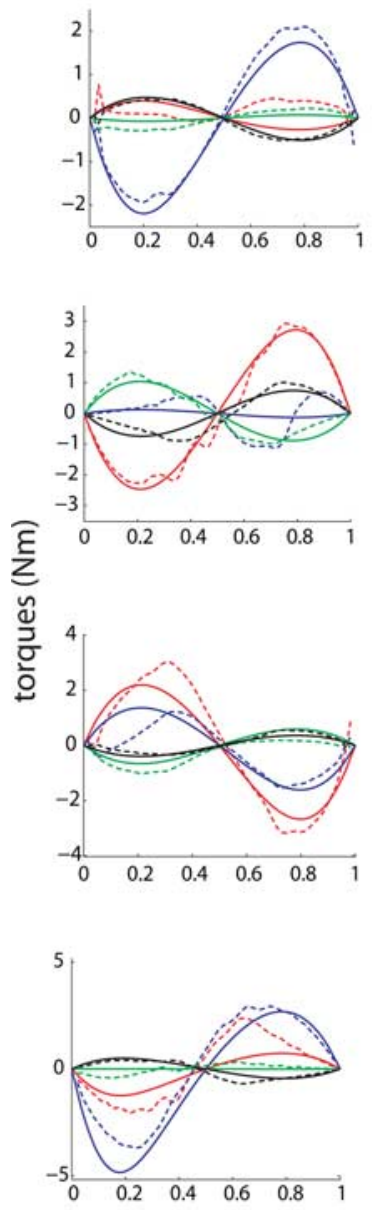

$\tau_{1}$
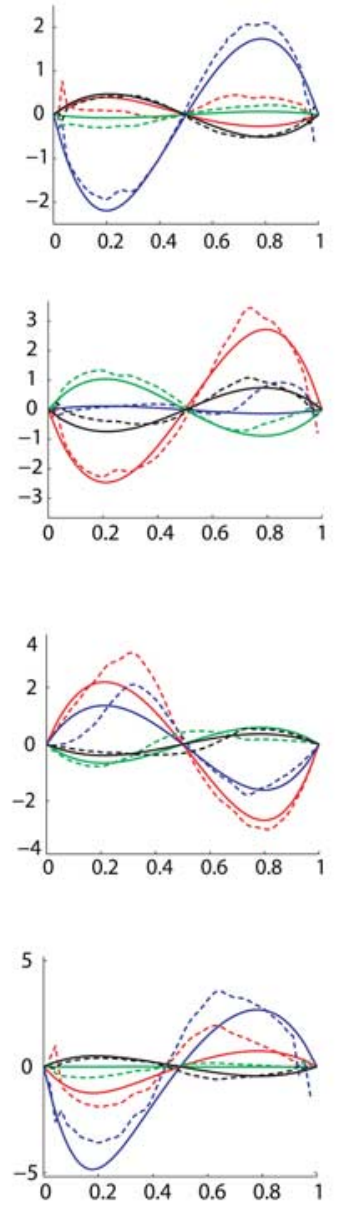

normalized time
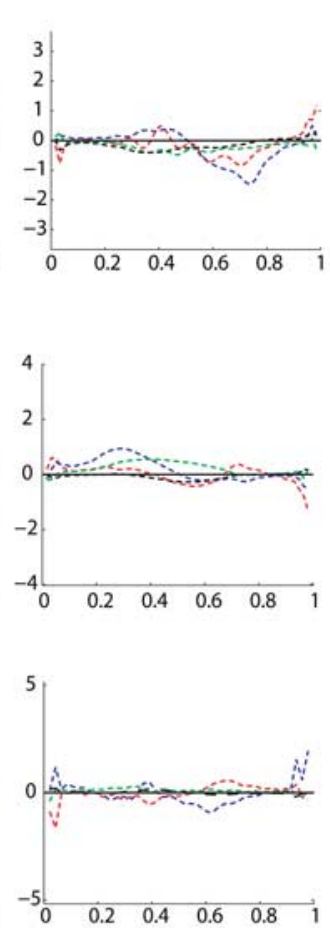

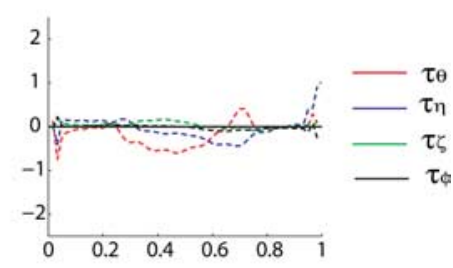

Within the framework of our model, however, the observed asymmetry in curvature for upward versus downward movements is caused by the different initial arm configurations in upward and downward movements and not by the presence of the gravitational field.

To conclude, we propose that the geometrical movement properties are acquired in non-Euclidean configuration space, independently from the temporal ones, based possibly on proprioceptive feedback, which leads the arm to reduce muscular effort. Once such properties are internalized, they may form part of a trajectory formation strategy that is implemented using feedforward control. Such a strategy integrates the two independent levels into a third spatiotemporal level. We showed that if the controller adopts such a solution, the arm joints move with near minimal torque change and minimal peak work while bringing the hand to the required location. Our data validated this assumption. In the light of these results, neurophysiologists may be encouraged to determine in more detail the specific brain regions involved in the space-time separation process during movement. Finally, our study showed the use of Riemannian metric spaces in the description of human arm movements, and it resulted ultimately in a computational model that reconciled seemingly opposing models contrasted in the literature.

\section{Appendix A}

The components of the elbow Jacobian for the four DOF linkage arm model defined by the joint angles $\boldsymbol{q}:=(\theta, \eta, \zeta, \phi)^{T} \in Q$ can be derived from the forward kinematic relations $\boldsymbol{x}_{e}=\boldsymbol{F}_{e}(\boldsymbol{q})$. The components of the elbow Jacobian are then defined as $J_{e, i j}$ $=\partial F_{e, i} / \partial q^{j},(i=1,2,3 ; j=1, \ldots, 4)$, leading to the nonzero components

$$
\begin{aligned}
& J_{e, 11}=-l_{u} \cos \theta \sin \eta \\
& J_{e, 12}=-l_{u} \cos \eta \sin \theta \\
& J_{e, 21}=l_{u} \cos \eta \cos \theta \\
& J_{e, 22}=-l_{u} \sin \eta \sin \theta \\
& J_{e, 31}=l_{u} \sin \theta,
\end{aligned}
$$

Similarly, for the hand Jacobian $J_{h, i j}=\partial F_{h, i} / \partial q^{j}$, we obtain the nonzero components

$$
J_{h, 11}=-\sin \eta\left[\left(l_{u}+l_{f} \cos \phi\right) \cos \theta-l_{f} \cos \zeta \sin \phi \sin \theta\right]
$$
bach, 1985; Papaxanthis et al., 2003). It has been concluded that this effect can be assigned to the gravitational field that acts, respectively, against and along the movement direction, respectively.

\section{Effect of gravity} torques. In previous studies, it has been reported that hand paths of movements in the sagittal plane tend to be more curved in the
upward than in the downward direction (Atkeson and Holler-

$$
\begin{aligned}
J_{h, 12} & =-\cos \eta\left[l_{f} \cos \theta \cos \zeta \sin \phi+\left(l_{u}\right.\right. \\
& \left.\left.+l_{f} \cos \phi\right) \sin \theta\right]+l_{f} \sin \eta \sin \phi \sin \zeta
\end{aligned}
$$




$$
\begin{aligned}
J_{h, 13} & =l_{f} \sin \phi(-\cos \eta \cos \zeta \\
& +\cos \theta \sin \eta \sin \zeta) \\
J_{h, 14} & =l_{f}[\sin \eta \sin \phi \sin \theta \\
& -\cos \phi(\cos \theta \cos \zeta \sin \eta \\
& +\cos \eta \sin \zeta)] \\
J_{h, 21} & =\cos \eta\left[\left(l_{u}+l_{f} \cos \phi\right) \cos \theta\right. \\
& \left.-l_{f} \cos \zeta \sin \phi \sin \theta\right] \\
J_{h, 22} & =-\sin \eta\left[l_{f} \cos \theta \cos \zeta \sin \phi+\left(l_{u}\right.\right. \\
& \left.\left.+l_{f} \cos \phi\right) \sin \theta\right] \\
& -l_{f} \cos \eta \sin \phi \sin \zeta \\
J_{h, 23} & =-l_{f} \sin \phi(\cos \zeta \sin \eta \\
& +\cos \eta \cos \theta \sin \zeta) \\
J_{h, 24} & =l_{f}[\cos \eta(\cos \phi \cos \theta \cos \zeta \\
& -\sin \phi \sin \theta)-\cos \phi \sin \eta \sin \zeta] \\
J_{h, 31} & =l_{f} \cos \theta \cos \zeta \sin \phi+\left(l_{u}\right. \\
& \left.+l_{f} \cos \phi\right) \sin \theta \\
J_{h, 33}= & -l_{f} \sin \phi \sin \theta \sin \zeta \\
J_{h, 34} & =l_{f}(\cos \theta \sin \phi+\cos \phi \cos \zeta \sin \theta),
\end{aligned}
$$

where $l_{u}$ and $l_{f}$ denote the length of the upper and forearm, respectively.

\section{Appendix B}

For a flexed arm and a fixed hand position $\boldsymbol{x}_{h}$, the arm can still rotate around an axis going through the shoulder and the fixed hand position (Fig. 1B). The allowed elbow positions are contained in a circle $C$ around this axis (Hollerbach, 1985). The circle can be obtained as the intersection between the sphere $S_{1}: x^{2}=l_{u}^{2}$ around the origin with radius $l_{u}$ and the sphere $S_{2}:\left(\boldsymbol{x}-\boldsymbol{x}_{h}\right)^{2}=l_{f}^{2}$ around the hand location $\boldsymbol{x}_{h}$ with radius $l_{f}$, giving the elbow location as a function of the rotation angle $\alpha \in[0,2 \pi)$ of the intersection circle $C=S_{1} \cap S_{2}$. After algebraic manipulation, the elbow location can be expressed as

$$
\boldsymbol{x}_{e}\left(\alpha, \boldsymbol{x}_{h}\right)=R_{z}(\varphi) R_{y}(\vartheta)\left[u \boldsymbol{e}_{x}+v \boldsymbol{e}_{r}(\alpha)\right]
$$

with the radial distance to the center of the intersection circle $u=(1 / 2 d)\left(l_{u}^{2}-l_{f}^{2}+d^{2}\right)$, intersection circle radius $v=\frac{1}{2 d} \sqrt{4 d^{2} l_{u}^{2}-\left(l_{u}^{2}-l_{f}^{2}+d^{2}\right)^{2}}$ and $d=\left|x_{h}\right|$. Furthermore, it is $\varphi=$ $\operatorname{atan} 2\left(y_{h}, x_{h}\right), \vartheta=\operatorname{asin}\left(z_{h} / d\right), \boldsymbol{e}_{x}=(1,0,0)^{T}, \boldsymbol{e}_{r}(\alpha)=(0, \cos \alpha, \sin$ $\alpha)^{T}$, where $\operatorname{atan} 2(a, b):=\operatorname{atan}(a / b)-\operatorname{sign}(a)[1-\operatorname{sign}(b)](\pi / 2)$. The angles $\alpha, \varphi$, and $\vartheta$ are defined in Figure $1 B . R_{y}, R_{z}$ define rotation matrices around the $y$ - and $z$-axes, respectively, and are given by $\tau_{1}$
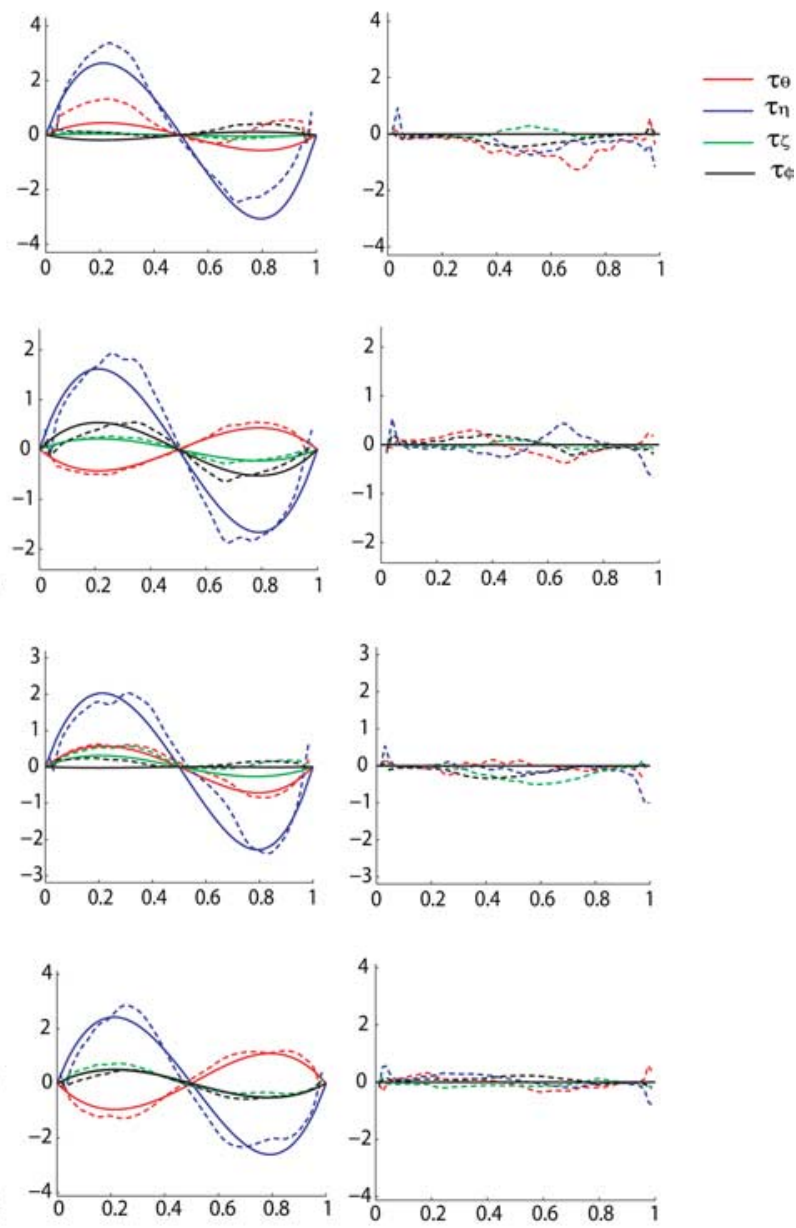

normalized time

Figure 17. Driving torques. Examples of predicted (solid) and measured (dashed) driving torques $\tau=\left(\tau_{q}, \tau_{\eta^{\prime}} \tau_{\zeta^{\prime}} \tau_{\phi}\right)^{T}$ in the frontoparallel movement condition. Description as in the radial condition.

$$
\begin{aligned}
& R_{y}(\vartheta) \\
& =\left(\begin{array}{ccc}
\cos \vartheta & 0 & -\sin \vartheta \\
0 & 1 & 0 \\
\sin \vartheta & 0 & \cos \vartheta
\end{array}\right), R_{z}(\varphi)=\left(\begin{array}{ccc}
\cos \varphi & -\sin \varphi & 0 \\
\sin \varphi & \cos \varphi & 0 \\
0 & 0 & 1
\end{array}\right) .
\end{aligned}
$$

\section{Appendix C}

The kinetic energy of a four DOF arm in terms of the coordinates $\mathbf{q}=(\theta, \eta, \zeta, \phi)^{T} \in Q$ is determined by

$$
\begin{aligned}
K= & \frac{1}{2}\left[I_{1}\left(\omega_{u, x}^{2}+\omega_{u, y}^{2}\right)+I_{2} \omega_{u, z}^{2}+I_{3}\left(\left(\omega_{u, y} \cos \phi+\omega_{u, z} \sin \phi\right)^{2}\right.\right. \\
& \left.+\left(\omega_{u, x}+\dot{\phi}\right)^{2}\right)+I_{4}\left(\omega_{u ; z} \cos \phi-\omega_{u, y} \sin \phi\right)^{2} \\
& \left.+2 A\left(\omega_{u, y}^{2} \cos \phi+\omega_{u, x}^{2} \cos \phi+\omega_{u, y} \omega_{u, z} \sin \phi+\omega_{u, x} \phi \cos \phi\right)\right]
\end{aligned}
$$

with

$$
\omega_{u}=[\dot{\eta} \sin \zeta \sin \theta+\dot{\theta} \cos \zeta, \dot{\eta} \cos \zeta \sin \theta-\dot{\theta} \sin \zeta, \dot{\eta} \cos \theta+\zeta]^{T}
$$


and the constants $I_{1}=I_{u, x}+m_{u} a_{u}^{2}+m_{f} l_{u}^{2}, I_{2}=I_{u, z}, I_{3}=I_{f, x}+$ $m_{f} a_{f}^{2}, I_{4}=I_{f, z}, A=m_{f} l_{u} a_{f}$ (Biess et al., 2001). The parameters $m_{i}$, $I_{i, x}, I_{i, y}, I_{i, z}, l_{i}, r_{i},(i=u, f)$ denote mass, principal moments of inertia around the $x$-, $y$-, and $z$-axes of the body-fixed coordinate systems, length, and distance to the center of mass of the upper and forearm, respectively. It was assumed that the (transversal) $x$ and $y$ components of the moment of inertia for the upper arm and forearm, respectively, are the same; i.e., $I_{u, x}=I_{u, y}$ and $I_{f, x}=$ $I_{f, y}$. The kinetic energy is a quadratic form in the joint velocities and can be written as

$$
K(\boldsymbol{q}, \dot{\boldsymbol{q}})=\frac{1}{2} \dot{\boldsymbol{q}}^{T} M(\boldsymbol{q}) \dot{\boldsymbol{q}}
$$

$M \in \mathbb{R}^{4 \times 4}$ is the kinetic energy metric (manipulator inertia matrix) with nonzero components

$$
M_{11}=I_{1}+2 A \cos \phi+I_{3} \cos ^{2} \zeta+I_{3} \cos ^{2} \phi \sin ^{2} \zeta+I_{4} \sin ^{2} \phi \sin ^{2} \zeta
$$

$$
M_{12}=-\left(\left(A+\left(I_{3}-I_{4}\right) \cos \phi\right) \cos \theta+\left(-I_{3}+\right.\right.
$$$$
\left.\left.I_{4}\right) \cos \zeta \sin \phi \sin \theta\right) \sin \zeta \sin \phi
$$

$M_{13}=-\left(A+\left(I_{3}-I_{4}\right) \cos \phi\right) \sin \phi \sin \zeta$

$M_{14}=\left(I_{3}+A \cos \phi\right) \cos \zeta$

$$
\begin{aligned}
M_{22}= & \frac{I_{1}}{2}-\frac{I_{1}}{2} \cos (2 \theta)+\cos ^{2} \theta\left(I_{2}+I_{3} \sin ^{2} \phi\right)+I_{4} \cos ^{2} \zeta \sin ^{2} \phi \sin ^{2} \theta \\
& +\cos ^{2} \phi\left(I_{4} \cos ^{2} \theta+I_{3} \cos ^{2} \zeta \sin ^{2} \theta\right)+A \cos \zeta \sin \phi \sin (2 \theta) \\
+ & \cos \phi\left(A-A \cos ^{2} \theta+A \sin ^{2} \theta+\left(I_{3}-I_{4}\right) \cos \zeta \sin \phi \sin (2 \theta)\right)
\end{aligned}
$$$$
+I_{3} \sin ^{2} \theta \sin ^{2} \zeta
$$

$M_{23}=\cos \theta\left(I_{2}+I_{4} \cos ^{2} \phi+I_{3} \sin ^{2} \phi\right)+\left(A+\left(I_{3}\right.\right.$

$$
\left.\left.-I_{4}\right) \cos \phi\right) \cos \zeta \sin \phi \sin \theta
$$

$M_{24}=\left(I_{3}+A \cos \phi\right) \sin \theta \sin \zeta$

$M_{33}=I_{2}+I_{4} \cos ^{2} \phi+I_{3} \sin ^{2} \phi$

$M_{44}=I_{3}$

and $M_{i j}=M_{j i}$.

\section{Appendix D}

The selection of the motion pattern on the temporal and geometrical as suggested in this work leads to the minimization of the peak value of kinetic energy and is therefore in accordance with the model suggested by Soechting et al. (1995). This result can be derived from our computational model as follows. For each path in configuration space, $\gamma_{\alpha}, \alpha \in I\left(\boldsymbol{x}_{f}\right)$, that connects a given initial arm configuration with the set of accessible final arm configurations compatible with a given hand location $\boldsymbol{x}_{f}$, the kinetic energy is given by the following (derived from Eq. 45):

$$
K(t, \alpha)=\frac{1}{2}\left(\frac{\sum(\alpha)}{\left|\boldsymbol{x}^{\prime}(\lambda, \alpha)\right|}\right)^{2} \dot{s}^{2}, \alpha \in I\left(\boldsymbol{x}_{f}\right),
$$

where $\Sigma$ is the total arc length of the path in configuration space, $\boldsymbol{x}^{\prime}(\lambda)$ denotes the derivative of the hand path, and $s$ is the Euclidean arc length of the hand path.

We determine next the single path out of the one-parameter family of geodesics that has minimal peak kinetic energy. The necessary condition for the peak value of the kinetic energy is

$$
\frac{d}{d t} K(t, \alpha)_{\mid t=t^{*}}=0
$$

where $t=t^{\star}$ denotes the peak time. If we assume that a minimum-jerk profile is imposed on the temporal level, Equation 98 leads to

$$
\tau^{\star}\left(1-\tau^{\star}\right)\left(1-2 \tau^{\star}\right)=0,
$$

resulting in $\tau^{\star}=1 / 2$, where $\tau$ is normalized time $\tau=t / T$. The peak of the kinetic energy thus occurs in the middle of the movement, which is in reasonable agreement with the experimental data (Figs. 9, 10). The peak value of the kinetic energy, $\hat{K}$, as a function of the parameter $\alpha$, follows then as

$$
\hat{K}(\alpha)=K\left(t^{*}, \alpha\right)=\frac{1}{2}\left(\frac{15 S(\alpha)}{8 T\left|\boldsymbol{x}^{\prime}(\lambda, \alpha)\right|}\right)^{2} \Sigma^{2}(\alpha), \alpha \in I\left(\boldsymbol{x}_{f}\right),
$$

where $S(\alpha)$ is the total length of the hand path corresponding to path $\gamma_{\alpha}$. For hand paths that are quasi-straight, as obtained in the computational model, it is $S(\alpha) /\left|\boldsymbol{x}^{\prime}(\lambda, \alpha)\right|=\int_{0}^{1}\left|\boldsymbol{x}^{\prime}(\lambda, \alpha)\right| \mathrm{d} \lambda / \mid \boldsymbol{x}^{\prime}(\lambda$, $\alpha) \mid \approx$ const, and the peak value of kinetic energy is proportional to the squared arc length of the path in configuration space. The geodesic with the shortest arc length that connects the initial and the final arm configurations results thus in the minimization of the peak value of kinetic energy.

\section{References}

Asada H, Slotine JJE (1986) Robot analysis and control. New York: Wiley. Atkeson T, Hollerbach JM (1985) Kinematic features of unrestrained vertical arm movements. J Neurosci 5:2318-2330.

Averbeck BB, Chafee MV, Crowe DA, Georgopoulos AP (2002) Parallel processing of serial movements in prefrontal cortex. Proc Natl Acad Sci USA 99:13172-13177.

Bengtsson SL, Ehrsson HH, Forssberg H, Ullen F (2005) Effectorindependent voluntary timing: behavioural and neuroimaging evidence. Eur J Neurosci 22:3255-3265.

Biess A, Flash T, Liebermann DG (2001) Multijoint point-to-point arm movements of humans in 3D-space: minimum kinetic energy paths. In: Proceedings of the Tenth Biennial Conference of the International Graphonomics Society (Meulenbroek RGJ, Steenbergen B, eds), pp 142-146. Nijmegen, The Netherlands: University of Nijmegen.

Biess A, Nagurka M, Flash T (2006) Simulating discrete and rhythmic multi-joint human arm movements by optimization of nonlinear performance indices. Biol Cybern 95:31-53.

Boessenkool JJ, Nijhof EJ, Erkelens CJ (1998) A comparison of curvatures of left and right hand movements in a simple pointing task. Exp Brain Res 120:369-376.

Buhusi CV, Meck WH (2005) What makes us tick? Functional and neural mechanisms of interval timing. Nat Rev Neurosci 6:755-765.

Desmurget M, Jordan M, Prablanc C, Jeannerod M (1997) Constraint and unconstraint movements involve different control strategies. J Neurophysiol 77:1644-1650.

do Carmo MP (1992) Riemannian geometry. Boston: Birkhäuser.

Flanders M, Hondzinski JM, Soechting JF, Jackson JC (2003) Using arm configurations to learn the effects of gyroscopes and other devices. J Neurophysiol 89:450-459.

Flash T (1987) The control of hand equilibrium trajectories in multi-joint arm movements. Biol Cybern 57:257-274.

Flash T, Hogan N (1985) The coordination of arm movements: an experimentally confirmed mathematical model. J Neurosci 5:1688-1703.

Flash T, Hollerbach JM (1982) Dynamic interactions between limb segments during planar arm movements. Biol Cybern 44:67-77.

Feldman AG (1966) Functional tuning of the nervous system with control 
of movement or maintenance of a steady posture. II. Controllable parameters of the muscle. Biophysics 11:565-578.

Feldman AG (1986) Once more on the equilibrium-point hypothesis (lambda model) for motor control. J Motor Behav 18:17-54.

Gamage SSHU, Lasenby J (2002) New least square solutions for estimating the average center of rotation and the axis of rotation. J Biomech 35:87-93.

Garraux G, McKinney C, Wu T, Kansaku K, Nolte G, Hallett M (2005) Shared brain areas but not functional connections controlling movement timing and order. J Neurosci 25:5290-5297.

Georgopoulos AP (1995) Motor cortex and cognitive processing. In: The cognitive neurosciences (Gazzaniga MS, ed), pp 507-517. Cambridge, MA: MIT.

Georgopoulos AP, Kettner RE, Schwartz AB (1988) Primate motor cortex and free arm movements to visual targets in three-dimensional space. II. Coding of the direction of movement by a neuronal population. J Neurosci 8:2928-2937.

Georgopoulos AP, Lurito JP, Petrides M, Schwartz AB, Massey JT (1989) Mental rotation of the neuronal population vector. Science 243:234-237.

Giakas G, Baltzopoulos V (1997) Optimal digital filtering requires a different cut-off frequency strategy for the determination of the higher derivatives. J Biomech 30:851-858.

Gribble PL, Ostry DJ (1999) Compensation for interaction torques during single- and multijoint limb movements. J Neurophysiol 82:2310-2326.

Harrington DL, Haaland KY, Hermanowicz N (1998) Temporal processing in the basal ganglia. Neuropsych 12:3-12.

Harris CM, Wolpert DM (1998) Signal-dependent noise determines motor planning. Nature 394:780-784.

Hermens F, Gielen CCAM (2004) Posture-based or trajectory-based movement planning: a comparison of direct and indirect pointing movements. Exp Brain Res 159:340-348.

Hollerbach JM (1985) Optimum kinematic design for a seven degree of freedom manipulator. In: Robotics research. The second international symposium (Hanafusa H, Inoue H, eds), pp 215-222. Cambridge, MA: MIT.

Ivry RB, Spencer MC (2004) The neural representation of time. Curr Opin Neurobiol 14:225-232.

Kathib O (1987) A unified approach for motion and force control of robot manipulators: the operational space formulation. IEEE J Robotic and Automation 3:43-53.

Kettner RE, Schwartz AB, Georgopoulos AP (1988) Primate motor cortex and free arm movements to visual targets in three- dimensional space. III. Positional gradients and population coding of movement direction from various movement origins. J Neurosci 8:2938-2947.

Lee DN, Port NL, Georgopoulos AP (1997) Manual interception of moving targets. II. On-line control of overlapping submovements. Exp Brain Res 116:421-433.

Liebermann DG, Biess A, Friedman J, Gielen CCAM, Flash T (2006) Intrinsic joint kinematic planning. I: Reassessing the Listing's law constraint in the control of three-dimensional arm movements. Exp Brain Res 171:139-154.

Moran DW, Schwartz AB (1999) Motor cortical activity during drawing movements: population representation during spiral tracing. J Neurophysiol 82:2693-2704.

Nakano E, Imamizu H, Osu R, Uno Y, Gomi H, Yoshioka T, Kawato M (1999) Quantitative examinations of internal representations for arm trajectory planning: minimum commanded torque change model. J Neurophysiol 81:2140-2155.

Nishikawa KC, Murray ST, Flanders M (1999) Do arm postures vary with the speed of reaching? J Neurophysiol 81:2582-2586.

Papaxanthis C, Pozzo P, Schieppati M (2003) Trajectories of arm pointing movements on the sagittal plane vary with both direction and speed. Exp Brain Res 148:498-503.

Rao SM, Harrington DL, Haaland KY, Bobholz JA, Cox RW, Binder JR (1997) Distributed neural systems underlying the timing of movements. J Neurosci 17:5528-5535.

Raymond JL, Lisberger SG, Mauk MD (1996) The cerebellum: a neuronal learning machine? Science 272:1126-1131.

Richardson MJE, Flash T (2002) Comparing smooth arm movements with the two-thirds power law and the related segmented-control-hypothesis. J Neurosci 22:8201-8211.

Sakai K, Hikosaka O, Takino R, Miyauchi S, Nielsen M, Tamada T (2000) What and when: parallel and convergent processing in motor control. J Neurosci 20:2691-2700.

Sakai BK, Ramnani N, Passingham RE (2002) Learning of sequences of finger movements and timing: frontal lobe and action-oriented representation. J Neurophysiol 88:2035-2046.

Sainburg RL, Poizner H, Ghez C (1993) Loss of proprioception produces deficits in interjoint coordination. J Neurophysiol 70:2136-2147.

Scott SH (2000) Population vectors and motor cortex: neural coding or epiphenomenon? Nat Neurosci 3:307-308.

Soechting JF, Buneo CA, Herrmann H, Flanders M (1995) Moving effortlessly in three dimensions: does Donders' law apply to arm movement. J Neurosci 15:6271-6280.

Sosnik R, Hauptmann B, Karni A, Flash T (2004) When practice leads to co-articulation: the evolution of geometrically defined movement primitives. Exp Brain Res 156:422-438.

Spencer RMC, Zelaznik HN, Diedrichsen J, Ivry RB (2003) Disrupted timing of discontinuous but not continuous movements by cerebellar lesions. Science 300:1437-1439.

Todorov E, Jordan MI (2002) Optimal feedback control as a motor coordination. Nat Neurosci 5:1226-1235.

Torres EB, Andersen R (2006) Space-time separation during obstacleavoidance learning in monkeys. J Neurophysiol 96:2613-2632.

Torres EB, Zipser D (2002) Reaching to grasp with a multi-jointed arm. I. Computational model. J Neurophysiol 88:2355-2367.

Torres EB, Zipser D (2004) Simultaneous control of hand displacements and rotations in orientation-matching experiments. J Appl Physiol 96:1978-1987.

Uno Y, Kawato M, Suzuki R (1989) Formation and control of optimal trajectories in human multijoint arm movements: Minimum torque-change model. Biol Cybern 61:89-101.

Wada Y, Kaneko Y, Nakano E, Osu R, Kawato M (2001) Quantitative examinations for multi joint arm trajectory planning — using a robust calculation algorithm of the minimum commanded torque change trajectory. Neural Netw 14:381-393.

Wang X, Maurin M, Mazet F, de Castro Maia N, Voinot K, Verriest JP, Fayet M (1998) Three-dimensional modelling of the motion range of axial rotation of the upper arm. J Biomech 31:899-908.

Xu D, Liu T, Ashe J, Bushara KO (2006) Role of the olivo-cerebellar system in timing. J Neurosci 26:5990-5995. 